\title{
Concevoir pour le pouvoir d'agir ensemble au sein des organisations : le cas d'un processus commercial
}

Designing for the power to act together in organizations: the case of a business process

Céline Poret, Viviane Folcher, Florence Motté and Yvon Haradji

\section{(2penEdition}

\section{Journals}

\section{Electronic version}

URL: http://journals.openedition.org/activites/2820

DOI: $10.4000 /$ activites.2820

ISSN: $1765-2723$

\section{Publisher}

ARPACT - Association Recherches et Pratiques sur les ACTivités

\section{Electronic reference}

Céline Poret, Viviane Folcher, Florence Motté and Yvon Haradji, « Concevoir pour le pouvoir d'agir ensemble au sein des organisations : le cas d'un processus commercial », Activités [Online], 13-2 | 2016, Online since 15 October 2016, connection on 01 May 2019. URL : http:// journals.openedition.org/activites/2820 ; DOI : 10.4000/activites.2820

This text was automatically generated on 1 May 2019.

\section{c)}

Activités est mis à disposition selon les termes de la licence Creative Commons Attribution - Pas d'Utilisation Commerciale - Pas de Modification 4.0 International. 


\title{
Concevoir pour le pouvoir d'agir ensemble au sein des organisations : le cas d'un processus commercial
}

\author{
Designing for the power to act together in organizations: the case of a
} business process

Céline Poret, Viviane Folcher, Florence Motté and Yvon Haradji

\section{EDITOR'S NOTE}

Article soumis le 02/02/2016, accepté le 30/06/2016

\section{Introduction}

1 Le changement de statut des entreprises, depuis un statut public où l'objectif est le service rendu au client, à un statut privé où l'objectif tient plus à l'inscription dans un univers concurrentiel, constitue toujours un bouleversement important pour la relation de service et les acteurs qui y sont engagés. Que ce soit pour le secteur des pompes funèbres (Trompette \& Boissin, 2000), La Poste (Flageul-Caroly, 2001) ou France Telecom (Hochereau, 2004), ces mutations d'entreprises publiques ont en effet toujours entraîné une rupture relativement au métier des acteurs qui se voient introduire des objectifs commerciaux pouvant entrer en contradiction avec les objectifs de service public, et qui développent alors des stratégies pour faire face aux conflits de logiques engendrés (Bernard, 2005; Buscatto, 2002 ; Calderon, 2006; Cihuelo, 2010; Flageul-Caroly, 2001). Suite à l'ouverture du marché de l'énergie à la concurrence, l'entreprise Electricité de France (EDF) a connu un tel bouleversement, entraînant notamment la transformation des organisations et de la relation de service, et impactant dès lors les métiers, les outils et l'organisation qui la composent. Dans ce cadre, des chercheurs en ergonomie de la Direction Recherche \& Développement (R\&D) de chez Électricité de France (EDF) ont 
initié un programme de recherche technologique nommé «Ingénierie pour la relation de service » dont l'objectif est de concevoir une relation de service de qualité en agissant sur les outils et sur l'organisation dans l'entreprise pour aider l'activité individuelle et collective et améliorer les conditions de réalisation du travail. La demande à l'origine de cette recherche s'inscrit dans ce programme de recherche et concerne la compréhension de la dynamique d'une activité collective qui est transverse à l'organisation et dont l'objectif est de produire le service au client. Cette compréhension de l'activité collective dite "activité collective transverse et transactionnelle» (Motté \& Haradji, 2010; Poret, 2015) doit permettre de contribuer à la conception des outils, de l'organisation et des métiers dans l'objectif de prendre le virage de l'inscription dans un univers concurrentiel en améliorant la satisfaction du client, les conditions de travail des agents, ainsi que l'efficacité de l'entreprise.

2 Après avoir présenté le paradigme de la transversalité dans lequel s'inscrivent les entreprises qui pensent désormais leur performance à partir de leurs processus opérationnels transversaux, nous argumentons que malgré ce changement de paradigme cette transversalité n'est pas réellement effective. Ce manque de transversalité réelle résulte d'une considération limitée de l'activité collective transverse et transactionnelle qui doit exister en dépassant les frontières classiques de l'organisation. Nous argumentons que concevoir pour cette activité collective consiste à concevoir pour le Pouvoir d'agir ensemble (Poret, 2015), qui implique de penser le rapport «ActeurEnvironnement». Nous présentons ensuite le type d'activité collective transverse et transactionnelle que nous avons étudié - un processus commercial de traitement de la demande de mise en service bi-énergie chez Électricité de France (EDF) - ainsi que notre méthodologie de recueil et d'analyse des données. Puis, nous décrivons les instruments qui ont été construits par les acteurs au service de cette activité collective - des instruments transitionnels coopératifs (Poret, ibid) - qui permettent la continuité malgré des organisations fragmentées et qui constituent les supports de la transversalité réelle au cœur des organisations. Nous proposons ensuite un critère pour la conception - le critère de continuité - dont la mobilisation lors de projets de conception portant sur l'environnement technico-organisationnel permet de prendre en considération la nécessité du développement d'une activité collective transverse et transactionnelle ainsi que de la construction et mobilisation d'instruments transitionnels coopératifs au service de cette activité.

\section{Un paradigme de la transversalité dans les entreprises}

\subsection{Une transformation de l'environnement économique}

Les organisations des entreprises ont connu un changement de paradigme, passant d'un paradigme jusqu'alors dominant de la verticalité et de la spécialisation des métiers à un paradigme de la transversalité (Poret, 2015) et de la coopération inter-métiers. Zarifian (1995) et Detchessahar (2003) parlent à ce propos du passage d'un "paradigme de la séparation des tâches et des responsabilités » à un " paradigme de la mise en coopération du travail». Ce changement de paradigme est fondé par une transformation de l'environnement économique datant de la fin des années 1970, début des années 1980 (Defelix, 1999; Du Tertre, 2013; Hammer \& Champy, 1993; Tarondeau, 2002; Veltz \& 
Zarifian, 1994 ; Watiez, 2002). D’une manière générale, cela a impliqué des besoins nouveaux pour l'organisation des entreprises, à qui les clients, la concurrence et le changement demandent désormais d'être flexibles (Greenan \& Mairesse, 2006 ; Hammer \& Champy, 1993; Roy \& Audet, 2002; Tarondeau, 2002; Watiez, 2002) et réactives (Askenazy, 2005), sous peine de ne pas survivre à cet environnement en perpétuel mouvement.

\subsection{Penser la performance à partir de la production de valeur pour un client : le processus, nouvel élément structurant des organisations}

4 Pour répondre à ces exigences de flexibilité et de réactivité, les entreprises ont transformé leurs organisations en redéfinissant leur performance à partir de leurs processus opérationnels transversaux. Nous sommes passés d'une entreprise pensée en termes de "râteau» à une entreprise pensée en termes d'"arête de poisson" (Ségrestin, 2004). L'idée générale de l'entreprise pensée en termes d'arête de poisson est constituée par « une ligne horizontale représentant le cours de l'activité [qui] traverse les structures verticales qui apportent leurs contributions» (Segrestin, ibid., p. 200). Cette arête symbolise le processus transversal, défini comme "ensembles coordonnés d'activités locales permettant, par leur conjonction, de produire de la valeur pour un client ou un élément de fonctionnement interne important» (Lorino, 2009). L'organisation n'est ici plus pensée selon une logique de spécialisation des métiers, comme c'est le cas pour la vision «râteau » de l'entreprise, mais selon une logique de production de valeur pour un client.

5 Aussi, ce changement paradigmatique fonde différentes transformations dans les entreprises :

- émergence de Nouvelles Formes Organisationnelles (NFO) comme les organisations en réseau, le management par projets, le développement de la polyvalence des agents du fait d'une compression des étapes constitutives du processus transversal sur une seule et même personne (Hammer, 1990);

- émergence de substrats techniques structurellement conçus autour de la notion de processus transversal, comme les Progiciels de Gestion Intégrés (PGI) ou Enterprise Resource Planning (ERP) (Mourlon \& Neyer, 2002) ainsi que les systèmes de workflow (Khoshafian \& Buckiewicz, 1998) ;

- émergence de nouveaux modèles managériaux comme le Lean Management (Lasnier, 2007 ; Ughetto, 2012), le management de la qualité avec la mise en œuvre de la norme ISO 9001 ainsi que le management de la performance et la mise en place d'indicateurs associés (Derujinsky-Laguecir, Kern, \& Lorino, 2011).

6 Toutefois, malgré ces transformations, la transversalité ne semble pas complètement effective, entraînant des conséquences pour la performance des entreprises, la satisfaction des clients et les conditions de travail des agents. En tant que client usager d'un service par exemple, nous avons déjà très certainement tous fait l'expérience de ce manque de transversalité rappelant sans cesse à chaque interlocuteur qui voulait bien l'entendre les raisons de notre appel, ce qui a été fait et dit depuis notre dernier appel, en raccrochant sans avoir obtenu de réponse et sans que le traitement de notre demande s'en trouve avancé... jusqu'à un prochain appel où il faudra refaire de même. En tant qu'agents impliqués dans la production d'un service, ceux qui sont au contact direct des 
clients sont souvent considérés comme redevables de la qualité de service, avec le poids d'une organisation sur les épaules (Motté \& Haradji, 2010). Cette position d'interface entre l'entreprise et le client peut être compliquée lorsque les agents n'ont pas les moyens de répondre aux demandes des clients. Les raisons de ce manque de transversalité réelle malgré les transformations de l'environnement technique, organisationnel et managérial des entreprises autour de la notion de processus transversal résident dans un présupposé qui considère que le processus serait égal à la somme des contributions individuelles qui le constituent. Or, bien plus que cela, le processus constitue une activité collective qui implique une coopération entre l'ensemble des acteurs qui y sont impliqués.

\subsection{Le processus, une activité collective transverse et transactionnelle}

7 L'ergonomie (Arnoud, 2013; Motté \& Haradji, 2010 ; Petit, 2005) et la gestion (Lorino, 2009, 2013 ; Lorino \& Peyrolle, 2005 ; Lorino \& Tricard, 2012) ont progressivement mis en évidence et conceptualisé cette nouvelle forme d'activité qui se déploie au cœur de ce processus transversal. Que cette activité collective soit nommée "activité collective transverse 》 (Motté \& Haradji, 2010) ou "activité collective conjointe» (Lorino, 2009, 2013 ; Lorino \& Peyrolle, 2005), il s'agit d'un même type d'activité collective qui se déploie de façon transverse à l'organisation. Il s'agit d'une activité collective « où des acteurs différents sont appelés à faire des choses différentes de manière coordonnée pour produire un résultat grâce à la complémentarité de leurs engagements " (Lorino \& Peyrolle, 2005, p. 224). Cette forme d'activité collective contraste avec celle plus classiquement appréhendée au sein des communautés de pratiques (Lave \& Wenger, 1991) où " un même type d'activité est exécuté par tous les membres d'un groupe » (Lorino \& Peyrolle, 2005, p. 223). Plus précisément, cette activité collective est transverse et transactionnelle :

- elle est transactionnelle ${ }^{1}$ dans le sens où les acteurs qui y sont engagés doivent parvenir à une transaction avec le monde: la production de valeur à destination d'un client. Cette transaction, «à l'instar des pièces d'un puzzle, ne prend sens qu'au niveau de la réunion de leurs actions respectives, au niveau de leur coopération» (Lorino, 2009; Lorino \& Peyrolle, 2005) ;

- elle est transverse dans le sens où pour parvenir à l'objectif final du processus - la transaction avec le monde - les différents acteurs engagés dans chacune des activités le constituant doivent coopérer en articulant leurs contributions respectives de façon transverse à l'organisation.

Elle se caractérise par quatre dimensions (Poret, 2015) :

- l'interdépendance entre les activités des différents acteurs qui y sont engagés : le résultat de l'activité de l'acteur 1 constitue le point de départ de l'activité de l'acteur 2, dont le résultat d'activité constituera à son tour le point de départ de l'acteur 3 et ainsi de suite ;

- le processus comme maille d'analyse : la maille d'analyse de cette nouvelle forme d'activité collective est constituée par le processus, car c'est au sein de ce processus - tourné vers la création de valeur pour un client - que cette activité collective se déploie et que les différents acteurs qui la constituent sont en interdépendance ;

- une interdépendance qui s'étale dans le temps : l'interdépendance des différentes activités constitutives de cette activité collective est une interdépendance qui s'étale dans le temps. Elle est ainsi distribuée temporellement, car les différents acteurs n'interviennent pas en 
même temps, mais à la suite les uns des autres, ainsi que spatialement, car ils appartiennent à des services/entités différents ne sont donc pas localisés au même endroit ;

- le système technico-organisationnel comme lieu de coordination : la coopération entre les acteurs doit nécessairement avoir lieu au sein du système technico-organisationnel, puisque ces acteurs ne partagent pas un même espace, ni un même temps. Nous utilisons le vocable «système technico-organisationnel » pour parler d'une forme d'organisation éclatée qui fragmente l'activité collective et d'un système technique qui est au cœur de cette

organisation, constitué notamment par un Progiciel de Gestion Intégré (PGI).

La mise en évidence de cette forme d'activité collective montre qu'à la vision transversale de l'entreprise correspondent des besoins pour la coopération transverse entre les acteurs d'un même processus. Boreham, Samurçay et Fischer. (2002) mettent ainsi en avant le «Work Process Knowledge » (WPK), qu'ils définissent comme un nouveau type de connaissance dont les acteurs réunis au sein d'un même processus doivent disposer s'ils veulent pouvoir coopérer au sein d'une organisation flexible. Il s'agit selon eux de prendre conscience des interdépendances entre les activités des différentes fonctions engagées dans un même processus, de comprendre les spécificités de chacune d'elles tout en comprenant comment le service est produit à un niveau systémique. Nascimento (2009) parle à ce titre d'un besoin de « la présence de tous dans l'activité de chacun » (p. 84).

\section{Ancrer la transversalité au cœur des organisations : concevoir pour le pouvoir d'agir ensemble}

\subsection{D'une non-considération à une considération limitée de l'activité collective transverse et transactionnelle}

10 À l'heure actuelle, cette activité collective est diversement considérée dans les organisations.

11 Elle est le plus souvent ignorée, la transversalité n'étant considérée qu'au travers des substrats techniques censés l'ancrer au cœur des organisations, comme les Progiciels de Gestion Intégrés (PGI). Le présupposé dominant derrière leur succès serait que leur seul déploiement suffirait pour que la transversalité soit effective. Ainsi, si chaque acteur engagé dans le processus réalise correctement la partie qui lui incombe alors, grâce à la dimension intégrée rendue possible par de tels substrats techniques, le tout serait égal à la somme des parties. Cette façon de considérer la transversalité ignore le fait qu'une coopération entre les acteurs du processus est nécessaire pour que cette dernière soit effective.

12 La mise en évidence de cette activité collective par l'ergonomie (Arnoud, 2013; Motté \& Haradji, 2010 ; Petit, 2005) et la gestion (Lorino, 2009, 2013 ; Lorino \& Peyrolle, 2005; Lorino \& Tricard, 2012) a eu pour conséquence une meilleure considération de cette dernière. Lorsqu'elle est considérée, la confrontation de pratiques entre les acteurs engagés dans un même processus transversal est une proposition récurrente pour favoriser l'émergence des conditions préalables à ce type d'activité collective (Arnoud, 2013; Arnoud \& Falzon, 2013, 2014 ; Lorino, 2009 ; Nascimento, 2009 ; Petit, 2005). La confrontation de pratiques permet en effet à chacun de ces acteurs de prendre conscience des interdépendances entre leurs contributions respectives. Cette prise de conscience concerne le fait qu'ils s'inscrivent dans un collectif dont ils ne soupçonnaient pas toujours 
l'existence, ainsi que l'identification des besoins des acteurs qui interviennent avec eux dans le cadre de ce collectif. Ils sont alors en mesure de mettre en œuvre ce qu'il faut pour répondre à ces besoins, c'est-à-dire à faire des choses qui ne sont pas directement pertinentes pour eux et pour leur contribution, mais qui s'adressent aux autres acteurs $\mathrm{du}$ collectif transverse et transactionnel. Par exemple, Lorino et Peyrolle (2005) montrent comment le fait de faire confronter les pratiques entre les acteurs d'un même processus - le processus « tarif vert » d'EDF-GDF - a permis aux vendeurs d'attribuer un sens à une facture dite "fantôme ", car nulle alors qu'ils n'en attribuaient aucun auparavant. Alors que cette dernière était indispensable pour les comptables, les vendeurs n'en voyaient pas l'intérêt et ne l'éditaient donc pas. La confrontation de pratiques entre ces acteurs a permis de faire prendre conscience de la nécessité d'éditer et d'envoyer une telle facture au client. Les vendeurs ont donc intégré ce besoin des comptables dans leur propre activité alors même que cela n'avait pas de plus-value pour leur propre contribution.

\subsection{Concevoir pour l'activité collective transverse et transactionnelle implique de penser le rapport "Acteur-Environnement »}

13 Si la prise de conscience des interdépendances permise par la confrontation de pratiques construit les conditions préalables à l'activité collective transverse et transactionnelle, elle ne garantit toutefois pas à tous les coups l'existence de cette activité en situation, c'est-à-dire dans le système technique et organisationnel dans lequel elle se développe. Que les acteurs du collectif transverse et transactionnel aient conscience et connaissance des besoins de chacun ne leur permettra pas de s'articuler concrètement dans un système technico-organisationnel qui ne le permettrait pas. Par exemple, supposons qu'un acteur 1 engagé dans le traitement de l'étape A (en front-office) ait connaissance des besoins de l'acteur 2 chargé de traiter l'étape B (en back-office) qui consisteraient en la transmission d'une référence que seul l'acteur 1 peut recueillir auprès du client. Il peut en avoir connaissance, et recueillir ladite référence auprès du client, mais si aucun espace d'échange entre l'acteur 1 et l'acteur 2 n'est prévu par le dispositif technicoorganisationnel alors cette connaissance restera vaine. Si la question de la transformation n'est envisagée qu'au travers de celle des acteurs du collectif transverse et transactionnel, sans se poser systématiquement celle de la transformation de l'environnement technicoorganisationnel qui constitue le lieu de leur coordination, alors l'approche de cette activité collective est limitée. Bien sûr, la transformation de l'environnement technicoorganisationnel n'est pas toujours nécessaire; la connaissance des besoins des autres acteurs du collectif transverse et transactionnel peut trouver un écho favorable dans cet environnement, rendant l'agir collectif possible. Elle peut également ne pas trouver cet écho favorable et ne rester qu'au niveau d'une connaissance indispensable pour agir collectivement, mais impossible à mobiliser concrètement en situation. Concevoir pour l'activité collective transverse et transactionnelle consiste ainsi à penser la question de la transformation relativement au couple « Acteur-Environnement » et non uniquement aux seuls acteurs engagés dans cette activité collective. Si cela est vrai pour tout projet de conception, cela l'est d'autant plus que la distribution temporelle et spatiale qui caractérise cette activité collective fait jouer un rôle charnière à l'environnement technico-organisationnel. 


\subsection{Concevoir pour l'activité collective transverse et transactionnelle : concevoir pour le Pouvoir d'agir ensemble}

14 Si la confrontation de pratiques et les transformations qu'elle permet ne sont pas systématiquement pensées dans un rapport avec l'environnement technicoorganisationnel, le risque est de permettre la seule construction de capacités d'agir au sens de Rabardel (2005a, 2005b), entendues en tant que moyens opératifs dont les acteurs disposent pour agir dans le Monde.

Dans le cadre conceptuel du Pouvoir d'agir mobilisé dans le présent travail (Rabardel, 2005a, 2005b), la distinction entre les capacités d'agir et les pouvoirs d'agir illustre la distinction entre le potentiel et l'actuel. Les capacités d'agir se trouvent du côté du potentiel et notre parti-pris est de les appréhender dans les termes d'instruments, c'est-àdire en tant que moyens opératifs construits par l'acteur pour agir dans le monde. Ce sont des capacités qui ne sont pas données, mais qui résultent d'un développement par et dans l'activité. Les pouvoirs d'agir se trouvent quant à eux du côté de l'actuel et sont en dépendance des circonstances, des conditions internes et externes de l'agir du sujet.

Nous proposons de nommer ces capacités d'agir concernant l'agir collectif au sein d'une activité collective transverse et transactionnelle des "capacités d'agir ensemble» (Poret, 2015). Si l'on souhaite que ces Capacités d'agir ensemble soient effectivement mobilisables en situation, c'est-à-dire qu'elles deviennent des "Pouvoirs d'agir ensemble » (Poret, ibid.), les conditions de leur mise en œuvre effective dans le localement et circonstanciellement situé doivent être pensées. Concevoir pour le Pouvoir d'agir ensemble des acteurs d'un collectif transverse et transactionnel implique de se poser la question des modes d'existence possible de cette activité collective en situation. Notre recherche a consisté à identifier les fondements du Pouvoir d'agir ensemble des acteurs d'un collectif transverse et transactionnel à partir de la mise en évidence des instruments subjectifs génériques (Rabardel, 1995) construits et mobilisés par les acteurs de ce collectif pour coopérer de façon transverse à l'organisation.

\subsection{Appréhender les fondements du Pouvoir d'agir ensemble : une ontologie phénoménologique}

17 À la suite de Theureau (2004, 2006, 2009, 2015), nous nous inscrivons dans une ontologie phénoménologique qui repose sur trois hypothèses :

- l'hypothèse de l'autonomie des systèmes vivants dite hypothèse générale de l'autopoïèse, formulée par Maturana \& Varela $(1980,1987)$. Selon cette hypothèse, un acteur - comme tout système vivant - constitue un système autonome, c'est-à-dire que son activité est construite à chaque instant par lui comme une interaction avec sa situation. Cette caractéristique d'autonomie signifie que l'interaction entre l'acteur et son environnement est asymétrique, dans le sens où l'acteur interagit seulement avec les caractéristiques de cet environnement qui sont pertinentes pour lui - ou pour sa dynamique interne - à chaque instant, et consiste en une dynamique de son couplage structurel avec cet environnement ;

- l'hypothèse de la conscience préréflexive qui s'inspire de l'œuvre philosophique de Sartre (1943, 1936/1972). La conscience préréflexive constitue un effet de surface de ces interactions asymétriques entre l'acteur et son environnement et accompagne toute activité humaine ; 
- une troisième hypothèse ontologique prolonge ces deux premières hypothèses vers l'activité collective, moyennant une référence supplémentaire à Sartre (1943) relativement au collectif entendu comme une "totalité détotalisée ". Selon cette troisième hypothèse ontologique, "la concaténation des activités individuelles-sociales constitue une activité collective qui est constamment décollectivisée par les acteurs individuels» (Sève, Theureau, Saury, \& Haradji, 2012).

Cette inscription dans une ontologie phénoménologique nous amène à considérer structurellement l'asymétrie des relations des acteurs avec leur environnement, et à conceptualiser l'activité collective comme une articulation d'activités individuelles. Il ne s'agit pas d'une ontologie subjectiviste qui isolerait l'individu et considèrerait l'activité collective comme une somme d'activités individuelles, ni d'une ontologie collectiviste qui exclurait cet individu, mais d'une ontologie qui considère structurellement l'asymétrie des relations entre l'individu et son environnement (autres acteurs y compris). De cette ontologie phénoménologique découle une conséquence épistémologique: le primat accordé au point de vue de l'acteur. En effet, la considération de la nature asymétrique des relations de l'Homme avec son environnement implique de passer par son point de vue pour connaître son activité. Les deux approches articulées dans le cadre de cette recherche - approche instrumentale (Folcher \& Rabardel, 2004a, 2004b ; Rabardel, 1995, 2005a, 2005b ; Rabardel \& Béguin, 2005 ; Rabardel, Folcher \& Béguin, 2002) et approche du cours d'action (Theureau, 2004, 2006, 2009; Theureau \& Jeffroy, 1994) - constituent toutes deux des approches "intrinsèques» (Daniellou \& Rabardel, 2005; Rabardel \& Béguin, 2005) de l'activité humaine, c'est-à-dire qui cherchent à "appréhender la réalité depuis le point de vue du sujet, et à comprendre les mécanismes d'engendrement de l'activité depuis cette base $»^{2}$ (Rabardel \& Béguin, ibid, p. 431). C'est la raison pour laquelle la compréhension des fondements du Pouvoir d'agir ensemble s'est faite à partir de l'analyse de l'activité individuelle-sociale (Theureau, 2004, 2006) des acteurs engagés dans une activité collective transverse et transactionnelle. Le passage par le point de vue individuel-social pour aller vers les dimensions collectives de l'activité humaine est fondé par nos choix ontologiques et épistémologiques.

\section{Analyser le traitement collectif de la demande du client}

\subsection{Différents types de demandes}

19 Notre terrain est constitué par les Centres de Relations Clients (CRC) d'Électricité de France (EDF). Il s'agit de centres d'appels où parviennent les appels des clients Particuliers qui souhaitent formuler une demande: ouvrir un contrat, demander un renseignement sur une facture, demander un plan de paiement... Cette entreprise compte 68 CRC répartis dans 8 régions, chacun d'eux traitant les demandes indépendamment de la localisation du client ${ }^{3}$.

20 Nous avons centré nos analyses sur l'activité des conseillers-clients, chargés de traiter les différentes demandes des clients. Ils sont engagés dans des activités de front-office qui consistent à répondre aux appels entrants des clients, et des activités de back-office qui consistent à traiter les courriers envoyés par ces derniers, résoudre des anomalies remontées par le système d'information, traiter des demandes évacuées temporairement vers une file d'attente (par eux-mêmes ou par d'autres) ainsi que des réclamations orales 
recueillies auprès des clients lors d'appels entrants. Ces différentes activités de front et de back-office sont soumises à la rémunération individuelle de la performance. L'examen des demandes formulées par les clients lors de notre recueil de données nous a permis de catégoriser ces demandes.

Figure 1 : Catégorisation des demandes formulées par téléphone/courrier.

Figure1: Categorization of demands formulated by phone/letters

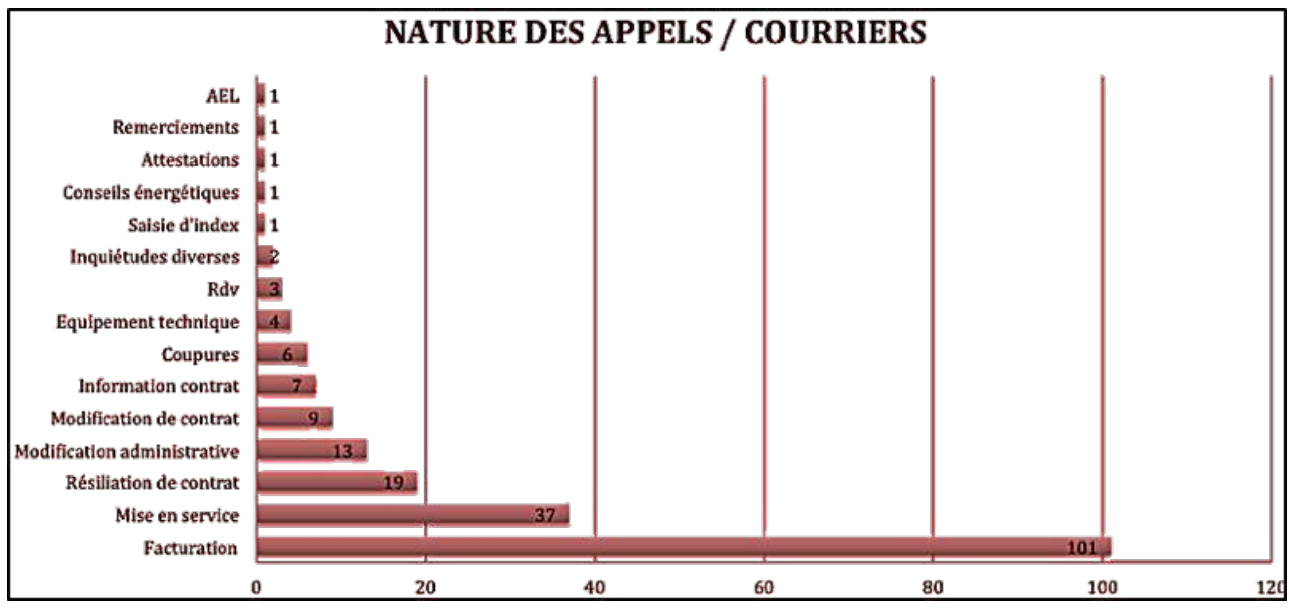

La figure 1 illustre la répartition de ces différentes catégories, basée sur l'ensemble des demandes traitées par les conseillers-clients lors de notre recueil de données. Les demandes liées à la facturation sont les plus nombreuses. Il s'agit par exemple d'un client qui appelle, car il a reçu le même échéancier de paiement que l'année précédente et souhaite savoir si c'est normal. Il peut également s'agir de clients qui ne comprennent pas leur facture et qui souhaitent avoir des explications, ou encore de clients qui ont eu des refus de prélèvement et qui souhaitent régulariser leur situation en payant leur facture. Pour la grande majorité de ces demandes, elles sont traitables immédiatement par les conseillers-clients qui renseignent les clients, prennent leur paiement en charge... Ce qui n'est pas le cas du second type de demandes le plus important : les demandes de mises en service ${ }^{4}$. Il s'agit de clients qui souhaitent ouvrir un contrat pour l'électricité seule ou pour l'électricité et le gaz. Le traitement de ces demandes n'est pas effectif immédiatement, c'est-à-dire que les conseillers-clients prennent les demandes des clients en considération, créent le contrat, mais le traitement effectif de ces demandes implique intrinsèquement d'autres étapes et d'autres compétences.

Ainsi, au-delà d'une catégorisation selon le thème de la demande, il est possible de les catégoriser selon leur caractère traitable en une seule fois (les demandes " one shot »), ou en plusieurs étapes. Les demandes " one shot " se caractérisent par un traitement en une seule fois, par un seul acteur, et par une superposition entre la fin du traitement de la demande et la fin du traitement de l'appel/courrier du client. Par exemple, dans le cadre du traitement d'une demande d'explication de facture, le conseiller-client analyse le compte du client et lui explique à quoi correspond le montant dû. Lorsque le client raccroche, sa demande est traitée. Les demandes intrinsèquement traitables en plusieurs étapes se caractérisent par un traitement nécessitant l'articulation de différentes étapes, impliquant plusieurs acteurs, dont les contributions respectives sont distribuées dans le temps et l'espace et par non-superposition entre la fin du traitement de la demande et la fin du traitement de l'appel/courrier du client. Lorsque le client raccroche, la demande 
est toujours « en cours » et d'autres contributions de la part d'autres acteurs, ailleurs et plus tard, sont attendues pour que le traitement de cette demande soit effectif.

Pour pouvoir être traitées de $\mathrm{A}$ à $\mathrm{Z}$, les demandes intrinsèquement traitables en plusieurs étapes impliquent - pour les différents acteurs engagés - de coopérer de façon transverse à l'organisation. Chacun est amené à traiter un «bout » de la demande et ainsi de suite jusqu'à ce que cette dernière soit effectivement traitée. C'est donc dans le cadre de ce type de demande que le développement d'une activité collective transverse et transactionnelle doit avoir lieu pour cette dernière soit effectivement et efficacement traitée.

\subsection{Le traitement de la demande de mise en service bi-énergie}

Les demandes de mise en service bi-énergie (électricité et gaz) correspondent à ce type de demandes, impliquant toujours au minimum 3 étapes pour être traitées jusqu'au bout dans les cas de figure où le traitement de la demande se déroule correctement, c'est-àdire en dehors des étapes résultant de traitements différés ou d'anomalies.

Figure 2 : Les étapes constitutives du traitement d'une demande de mise en service bi-énergie. Figure 2: The constituent stages of processing a request to put electricity and gas into service

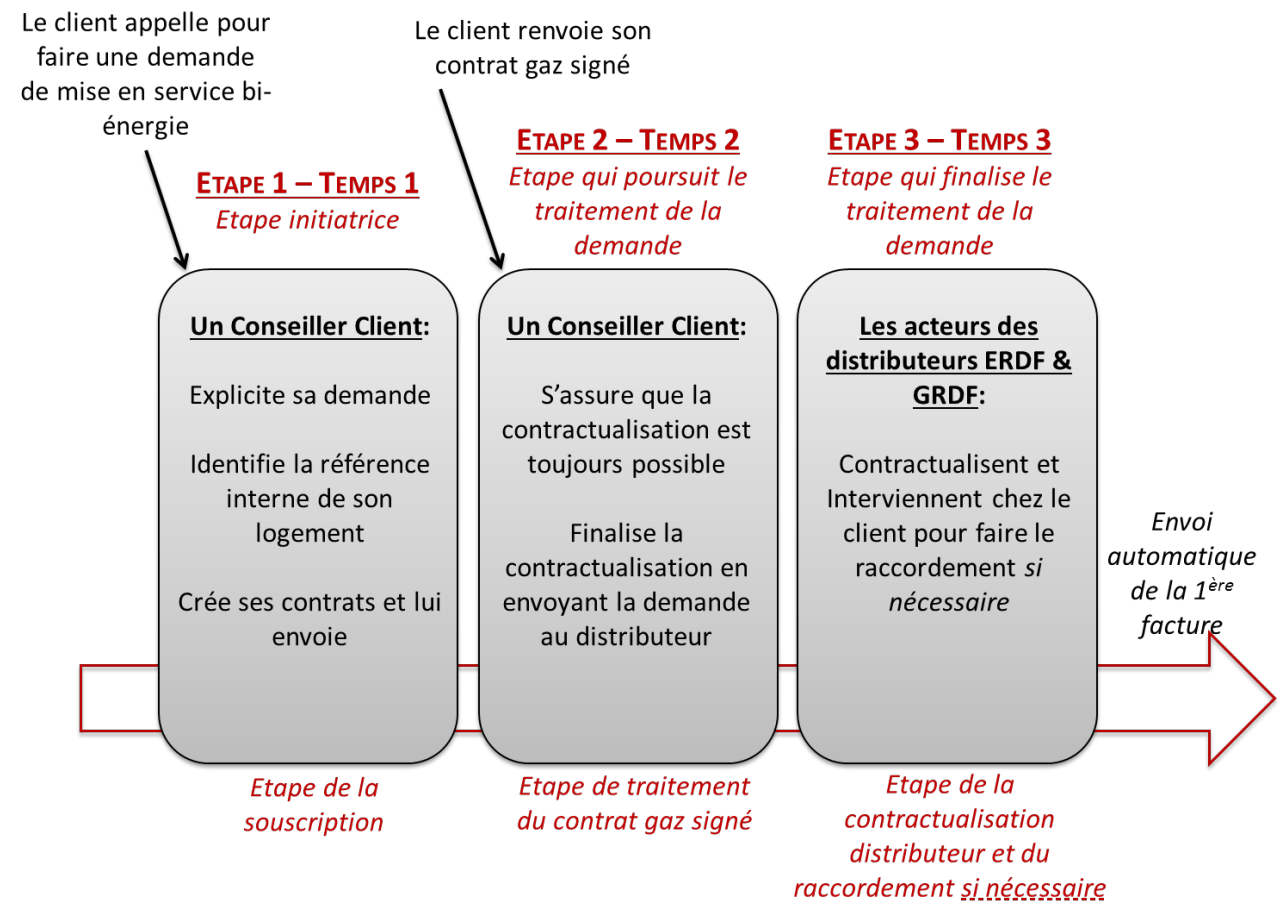

La figure 2 illustre les étapes constitutives du traitement de ce type de demandes. Ainsi, l'étape $\mathrm{n}^{\mathrm{o}} 1$ consiste à prendre la demande du client en considération, à l'expliciter, à créer les contrats et à les envoyer au client afin qu'il les renvoie signés. Une fois que le client a renvoyé son contrat gaz signé, l'étape $\mathrm{n}^{\circ} 2$ consiste à s'assurer que la poursuite de la contractualisation est possible, et à finaliser la contractualisation du client en envoyant la demande aux distributeurs afin qu'ils fassent le nécessaire. Enfin, l'étape $\mathrm{n}^{\mathrm{0}} 3$ consiste à intervenir chez le client pour le raccordement. L'envoi automatique de la première facture signe la fin du traitement de la demande de mise en service bi-énergie. Le conseiller-client qui est chargé de traiter le contrat gaz signé d'un client dans le cadre 
d'une demande de mise en service bi-énergie (étape $\mathrm{n}^{\mathrm{Q}} 2$ ) s'inscrit donc dans une demande qui a déjà été initiée par un autre conseiller-client qui avait créé le contrat, et qui va se poursuivre au-delà de sa propre contribution avec l'intervention des distributeurs chez le client. Autrement dit il s'inscrit dans le traitement d'une demande qui a un passé et un avenir. Avec lui, l'ensemble des acteurs qui sont intervenus et qui interviendront ponctuellement dans le traitement de la demande sont engagés dans une activité collective transverse et transactionnelle.

\subsection{Méthodologie de recueil des données}

D'un point de vue méthodologique l'objectif du recueil de données est donc de pouvoir documenter la conscience préréflexive des acteurs, c'est-à-dire le «montrable, racontable, commentable à tout instant » sans ruiner l'activité qui est en cours afin de ne pas briser la dynamique du cours d'action. La méthodologie de recueil de données qui permet cela, explicitée par Theureau $(2004,2006)$, consiste à relier des observations et des enregistrements en continu du comportement des acteurs, des verbalisations provoquées des acteurs en situation et des verbalisations en autoconfrontation. Notre recueil de données a ainsi consisté à réaliser des enregistrements audio-vidéo en continu de l'activité des conseillers-clients engagés dans le traitement des différentes étapes constitutives de la demande de mise en service bi-énergie. Nous étions assis à côté du conseiller-client, caméra centrée sur leur écran d'ordinateur et prenions des notes sur le déroulement de leur activité ainsi que sur les verbalisations du client qui était en doubleécoute. Immédiatement après le traitement de l'étape par le conseiller-client nous revenions avec lui sur ce qu'il venait de faire, dans le cadre d'entretiens nommés « posttraitement du cas » qui avaient le même objectif que les entretiens d'autoconfrontation (Theureau, 2004, 2006), à savoir l'expression de la conscience préréflexive de l'acteur. La spécificité de l'activité collective considérée dans le cadre de ce travail nous a amenés à assigner un second objectif à ces entretiens : revenir avec les acteurs sur l'avant et l'après de leur propre contribution afin de nous permettre de situer plus largement cette contribution dans la continuité du traitement de la demande. En effet, une des contraintes intrinsèques de notre terrain est constituée par le fait qu'appréhender le traitement d'une seule et même demande concernant un seul et même client de $\mathrm{A}$ à $\mathrm{Z}$ est impossible. Il est en effet impossible d'anticiper le moment où le client va renvoyer son contrat, le moment où ce contrat va être numérisé par l'acteur chargé de le faire ainsi que le Centre de Relations Clients (CRC) où ce contrat numérisé sera réceptionné et dispatché pour traitement à un Conseiller Client. Pour pallier à cette contrainte, nous avons assigné un second rôle à ces entretiens : en plus de permettre l'accès à la conscience préréflexive des acteurs pour le traitement de l'étape actuelle nous les avons utilisés pour revenir avec l'acteur sur l'avant et l'après de sa propre contribution. Cela nous a permis de situer plus largement sa contribution dans la continuité du traitement de la demande de mise en service bi-énergie.

En tout, nous avons recueilli 95h d'enregistrement audio-vidéo, auprès de 24 conseillersclients et 2 de leurs responsables hiérarchiques directs. 


\subsection{De l'individuel au collectif, du Pouvoir d'agir au Pouvoir d'agir ensemble}

Concernant l'analyse de ces données, et au regard de la conceptualisation de l'activité collective qui découle de nos choix ontologiques et épistémologiques, l'objectif est d'appréhender activité individuelle et activité collective en un seul mouvement. Cela a consisté à passer nécessairement par le point de vue individuel de chaque acteur engagé dans cette activité collective, point de vue que l'on peut qualifier d'individuel-social dans le sens où il ouvre sur l'activité des autres acteurs qui est prise en compte pour agir. Plus précisément, nous avons analysé finement l'activité individuelle-sociale de chaque acteur et examiné dans quelle mesure cette activité était structurée par une activité collective de type transverse et transactionnelle. Ainsi, le point de départ de nos analyses était constitué par la construction d'un graphe d'analyse modélisant la construction pas à pas de l'activité individuelle-sociale de chaque conseiller-client, à partir de la mise en évidence des différentes préoccupations ${ }^{5}$ qui structurent cette activité.

Par exemple, un conseiller-client est engagé dans le traitement d'une étape de souscription, première étape du traitement de la demande de mise en service. Alors qu'il a identifié la référence du logement du client lui permettant de créer le contrat - le point de livraison -, il s'aperçoit que ce dernier n'a pas toutes les informations sur le logement dont il aura besoin pour créer ce contrat. Il s'apprête donc à raccrocher avec le client, mais avant cela il s'engage :

- dans une séquence ${ }^{6}$ au cours de laquelle il conseille le client sur les modalités de son rappel: cela consiste à lui indiquer les informations dont il aura besoin lors du rappel, à lui communiquer certaines références que le client devra donner lorsqu'il rappellera, afin que le conseiller-client qu'il a en ligne l'identifie rapidement, ainsi qu'à répondre à différentes questions du client sur les pièces dont il devra disposer lors de son rappel ;

- dans une séquence au cours de laquelle il crée un "contact», c'est-à-dire un objet informatique destiné à rendre compte de la nature de l'appel du client tout en y laissant un commentaire.

Les préoccupations qui fondent ces séquences sont :

- " atteindre ses objectifs de performance relativement à cette demande de mise en service »: en effet, la création de l'objet informatique « contact » est comptabilisée dans le cadre d'un objectif de traçage des demandes traitées;

- «articuler son activité à celle des autres acteurs du traitement de la demande»: en conseillant le client sur les modalités de son rappel et en lui donnant les références de son dossier le conseiller-client prépare la reprise de ce traitement par celui ou celle qui aura le client en ligne lorsqu'il rappellera avec toutes les informations. Aussi, si la création de l'objet informatique « contact» est fondée par la préoccupation d'atteindre ces objectifs de performance, le fait d'y renseigner un commentaire n'est pas fondé par la même préoccupation. En faisant cela, le conseiller-client prépare la reprise ultérieure du traitement de cette demande par celui ou celle qui aura le client en ligne lors de son rappel. La figure 3 illustre cette préoccupation « articuler son activité à celle des autres acteurs du traitement de la demande ", relativement au fait de laisser un commentaire dans le contact. 
Figure 3 : Illustration de la préoccupation « articuler son activité à celle des autres acteurs du traitement de la demande ".

Figure 3: Illustration of the preoccupation "to link one's own activity with that of the other actors involved in request processing"

"Le fait de mettre le contact [le commentaire dans le contact], la personne qui va reprendre le dossier demain avec cette référence verra le contact et dedans j'ai mis le point de livraison. Comme ça elle n'aura pas besoin de refaire les recherches »

À partir des différentes modélisations de l'activité individuelle-sociale singulière des conseillers-clients nous avons pu accéder:

- aux dimensions singulières et génériques de l'activité des conseillers-clients: la comparaison de l'ensemble des modélisations singulières de l'activité individuelle-sociale des conseillers-clients nous a permis de monter en généralisation et d'identifier des préoccupations typiques qui se retrouvent dans chacune de ces modélisations. Par exemple, la préoccupation «articuler son activité à celle des autres» se retrouve dans l'activité de chacun des conseillers-clients engagés dans le traitement d'une étape de la demande de mise en service bi-énergie ;

- aux instruments génériques construits par les acteurs entendus en tant que Capacités d'agir : l'examen des ressources artefactuelles systématiquement mobilisées au service d'une préoccupation typique nous a permis d'identifier les instruments génériques (Rabardel, 1995) construits par les acteurs au service de leur activité, c'est-à-dire leurs Capacités d'agir (Rabardel, 2005a, 2005b) ;

- aux dimensions individuelles et collectives de l'activité des conseillers-clients: la modélisation de l'ensemble des préoccupations qui structurent l'activité individuelle-sociale des acteurs montre que ces dernières peuvent concerner leur activité individuelle ou tendre vers une activité collective. Par exemple, la préoccupation «atteindre ses objectifs de performance» concerne l'activité individuelle tandis que la préoccupation « articuler son activité à celle des autres » montre une structuration de l'activité individuelle par une activité collective de type transverse et transactionnelle. La mise en évidence des instruments génériques construits au service de cette préoccupation typique qui tend vers l'activité collective transverse et transactionnelle nous a permis de passer de la documentation de la Capacité d'agir à celle de la Capacité d'agir ensemble ;

- aux entraves à la mobilisation de ces Capacités d'agir ensemble : à partir de la connaissance des Capacités d'agir ensemble des acteurs, c'est-à-dire des instruments génériques qu'ils ont construits au service de l'activité collective transverse et transactionnelle, nous avons pu identifier les circonstances qui rendent leur mobilisation possible, complexifiée ou impossible. Ces éléments nous ont ainsi permis d'identifier les fondements du Pouvoir d'agir ensemble.

Ainsi, le passage par une phénoménologie de l'activité humaine nous a permis de partir d'une modélisation de l'activité individuelle-sociale de chaque conseiller-client et d'aller vers la mise en évidence de différentes dimensions de cette activité, dont sa dimension collective. Ce faisant, le passage par une phénoménologie de l'activité humaine nous a permis de passer du Pouvoir d'agir au Pouvoir d'agir ensemble. 


\subsection{Le client, acteur du traitement de sa demande}

Le traitement de la demande de mise en service bi-énergie se construit à la fois dans l'entreprise, par l'intermédiaire des interactions transverses qu'il implique entre les acteurs, ainsi que chez le client qui a formulé la demande et qui joue un rôle actif dans son traitement. Jusqu'à présent, les études ergonomiques dans le domaine de la relation de service ont principalement privilégié l'analyse des interactions synchrones entre les clients et les acteurs professionnels engagés dans le traitement de leur demande (Cerf, Valléry \& Boucheix, 2004), se centrant alors sur la dimension «transactionnelle » de l'activité collective. Nous avons fait le choix de nous centrer sur sa dimension «transverse » en partant du point de vue des conseillers-clients afin de comprendre les mécanismes de coordination qu'ils mettent en œuvre pour coopérer avec les autres acteurs engagés dans le traitement de la demande de mise en service bi-énergie. De ce choix résultent des analyses où le client est présent, mais appréhendé au travers du point de vue des conseillers-clients. Cela peut donner l'impression qu'il constitue un élément résiduel de notre travail, voire qu'il a été oublié. Cela n'est pas le cas, mais le primat accordé au point de vue de l'acteur aurait impliqué d'articuler notre analyse de cette activité collective du point de vue des conseillers-clients à une analyse faite du point de vue des clients. Cela n'a pas été réalisé dans le cadre de ce travail, mais dans le cadre de la poursuite du programme de recherche «Ingénierie pour la relation de service » au sein duquel cette recherche s'inscrit chez EDF R\&D.

\section{Les instruments transitionnels coopératifs au service de la continuité du traitement collectif de la demande du client}

\subsection{Les capacités d'agir ensemble : entre enquête et mise en archives}

Pour coopérer dans le cadre d'une activité collective transverse et transactionnelle, les conseillers-clients articulent, à chaque étape : reconstruction de dimensions passées du traitement de cette demande, traitement de l'étape actuelle et anticipation du futur de ce traitement. Coopérer au sein de ce type d'activité collective consiste donc à articuler passé-présent-futur du traitement de la demande à chaque étape (Figure 4). 
Figure 4 : Coopérer au sein d'une activité collective transverse et transactionnelle : articuler passéprésent-futur à chaque étape.

Figure 4: Cooperating in a transversal and transactional collective activity: connecting past-presentfuture at each stage

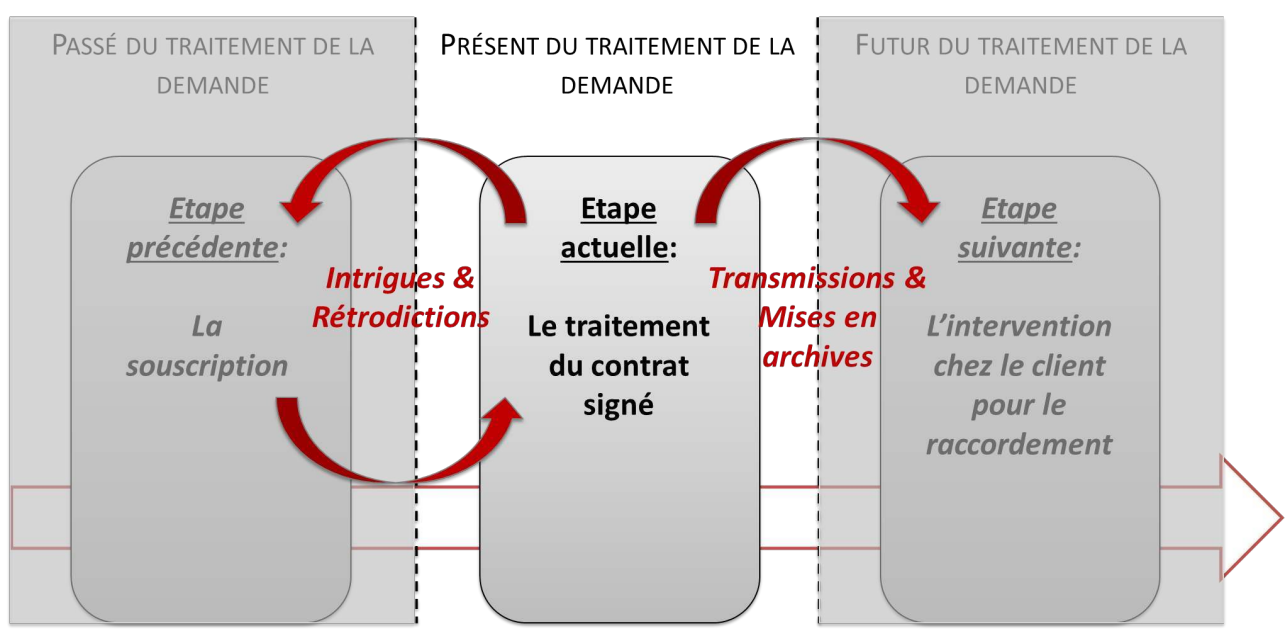

Un conseiller-client qui s'engage dans le traitement d'une demande de mise en service biénergie a besoin de comprendre ce qu'il s'est passé pour ce traitement jusqu'à l'instant $t$. Pour cela, il s'engage dans une activité d'enquête dont le résultat doit lui permettre de reconstruire le cours temporel des évènements pour le traitement de cette demande, expliquant l'état dans lequel il la trouve. Il s'engage alors dans une intense activité interprétative: il s'agit de "rechercher, consulter, vérifier, faire des hypothèses, préciser... ». À l'instar des historiens, les conseillers-clients s'engagent dans la résolution d'« intrigues » (Veyne, 1971) différentes selon l'étape qu'il s'agit de traiter. Par exemple, un conseiller-client qui s'engage dans le traitement d'une étape de traitement du contrat gaz signé (étape $\mathrm{n}^{\mathrm{N}} 2$ ) doit résoudre l'intrigue "La contractualisation est-elle toujours possible? » avant de s'engager dans l'étape qui lui incombe en tant que telle. Pour cela, il va rechercher dans les différents outils à sa disposition des indices qui lui permettent de reconstruire l'histoire du traitement de cette demande du point de vue de la contractualisation jusqu'à l'instant $t$. De même, un conseiller-client qui s'engage dans le traitement d'une étape supplémentaire par rapport à celles prévues résultant d'une anomalie doit résoudre l'intrigue "Qu'est-ce qui a mené à cette anomalie?» pour comprendre ce qu'il faut faire pour traiter cette étape. Il s'engage alors dans une activité d'enquête où il cherche, sélectionne différents éléments qui lui permettent de reconstruire l'histoire du traitement de cette demande jusqu'à l'instant $t$, qui pourrait expliquer son état actuel. L'extrait ci-après (Figure 5) illustre ce processus d'enquête dont l'objectif est de résoudre l'intrigue "Qu'est-ce qui a mené cette demande en anomalie ?». La partie qui est en gras montre que, comme les historiens, les conseillers-clients sont amenés à faire des "rétrodictions» (Veyne, 1971), c'est-à-dire à faire des inférences raisonnables sur ce qui a pu se passer afin de combler des sources lacunaires, afin de «boucher les trous » (Veyne, ibid, p. 194). 
Figure 5 : Extrait de protocole relativement à l'intrigue «Qu'est-ce qui a mené cette demande en anomalie?".

Figure 5: Extract from a protocol concerning the "What turned this request into an anomaly ?"

" Donc là il y a un contact qui dit qu'effectivement index donnés beaucoup trop élevés... Rappelle ce soir pour donner rendez-vous ou confirmer rendez-vous de relève spéciale... Donc en fait ça ce ne sont pas les procédures. Ne jamais faire un contrat sans faire une demande

via ERDF. La personne elle a fait une demande qui est revenue sûrement avec un smiley rouge et puis bah elle n'a pas continué, elle l'a laissée comme ça en attendant que le client il rappelle. Le problème c'est que souvent les clients ils rappellent pratiquement jamais ou le client comme il a l'électricité après il est pris par d'autres choses et puis il s'ne fiche en fait. Comme là..."

Pour résoudre ces intrigues qui caractérisent la prise de relais dans une activité collective transverse et transactionnelle, les conseillers-clients ont construit des instruments dans le cours de leurs expériences professionnelles et à l'occasion de genèses. Ces instruments constituent leurs Capacités d'agir. Ces Capacités d'agir, qui sont des Capacités d'enquête, articulent trois instruments ${ }^{7}$ :

- un instrument de prise de connaissance de l'instant $t:$ il permet de saisir rapidement l'état du traitement de la demande à l'instant $t$, selon l'étape et donc l'intrigue dans laquelle on est engagé. Il s'agit pour le conseiller-client de consulter, de rechercher, d'accéder à différents endroits du système d'information et/ou de questionner le client lorsque cela est possible. Les ressources artefactuelles mobilisées dans cet instrument sont constituées par des synthèses (Figure 6) relatives au client ou au logement concerné - chez EDF et chez le distributeur -, ainsi que par différentes listes (Figure 6) relatives aux contrats rattachés à un client, aux contrats rattachés à un logement, aux motifs de contacts d'un client ;

Figure 6 : Illustration de synthèses (à gauche) et de listes (à droite), ressources artefactuelles de l'instrument de prise de connaissance de l'instant $t$

Figure 6: Illustration of overviews and lists, artefactual resources constituent of the "understanding at time $t^{\prime \prime}$ instrument

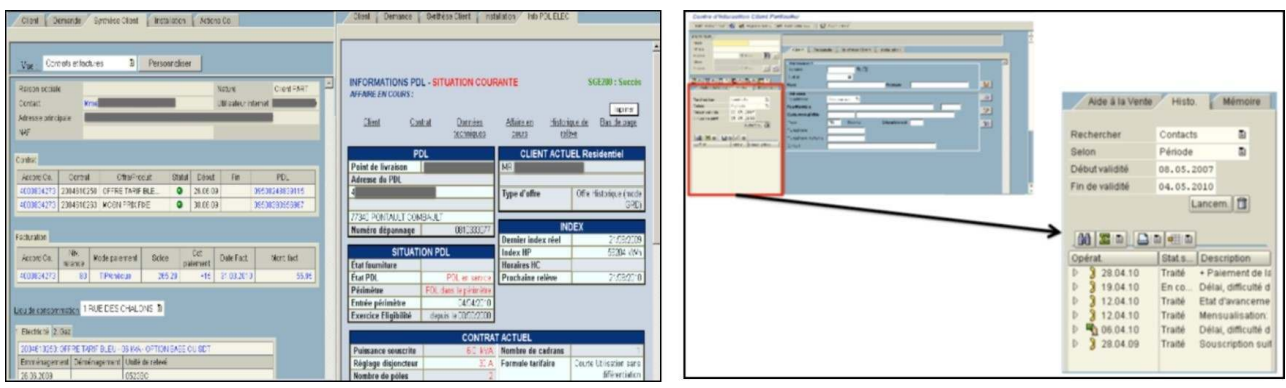

- un instrument de synthèse historique : il permet de resituer l'état dans lequel on trouve la demande à l'instant $t$ dans une histoire, c'est-à-dire de reconstruire le cours des évènements et actions des autres acteurs qui sont intervenus en amont sur le traitement de cette 
demande. Il s'agit pour le conseiller-client de consulter, de rechercher, d'accéder à différents endroits du système d'information et/ou de questionner le client lorsque cela est possible. Les ressources artefactuelles mobilisées dans cet instrument sont constituées par les commentaires (Figure 7) qui ont été laissés à différents endroits du système d'information par les acteurs qui ont précédé dans le traitement de la demande ;

- un instrument de recherche d'une information historique : il permet de rechercher une information précise relative au passé du traitement de la demande dont on a besoin pour poursuivre ce traitement. Comme pour les deux autres instruments constitutifs des Capacités d'enquête il s'agit pour le conseiller-client de rechercher, d'accéder à différents endroits du système d'information et/ou de questionner le client lorsque cela est possible. Les ressources artefactuelles mobilisées dans cet instrument sont constituées par les commentaires (Figure 7) qui ont été laissés à différents endroits du système d'information par les acteurs qui ont précédé dans le traitement de la demande.

Figure 7 : Les commentaires, une ressource artefactuelle constitutive des instruments de synthèse historique et de recherche d'une information historique

Figure 7: Comments, an artefactual resource constituent of the "historical overview" instrument and of the "searching for historical information" instrument

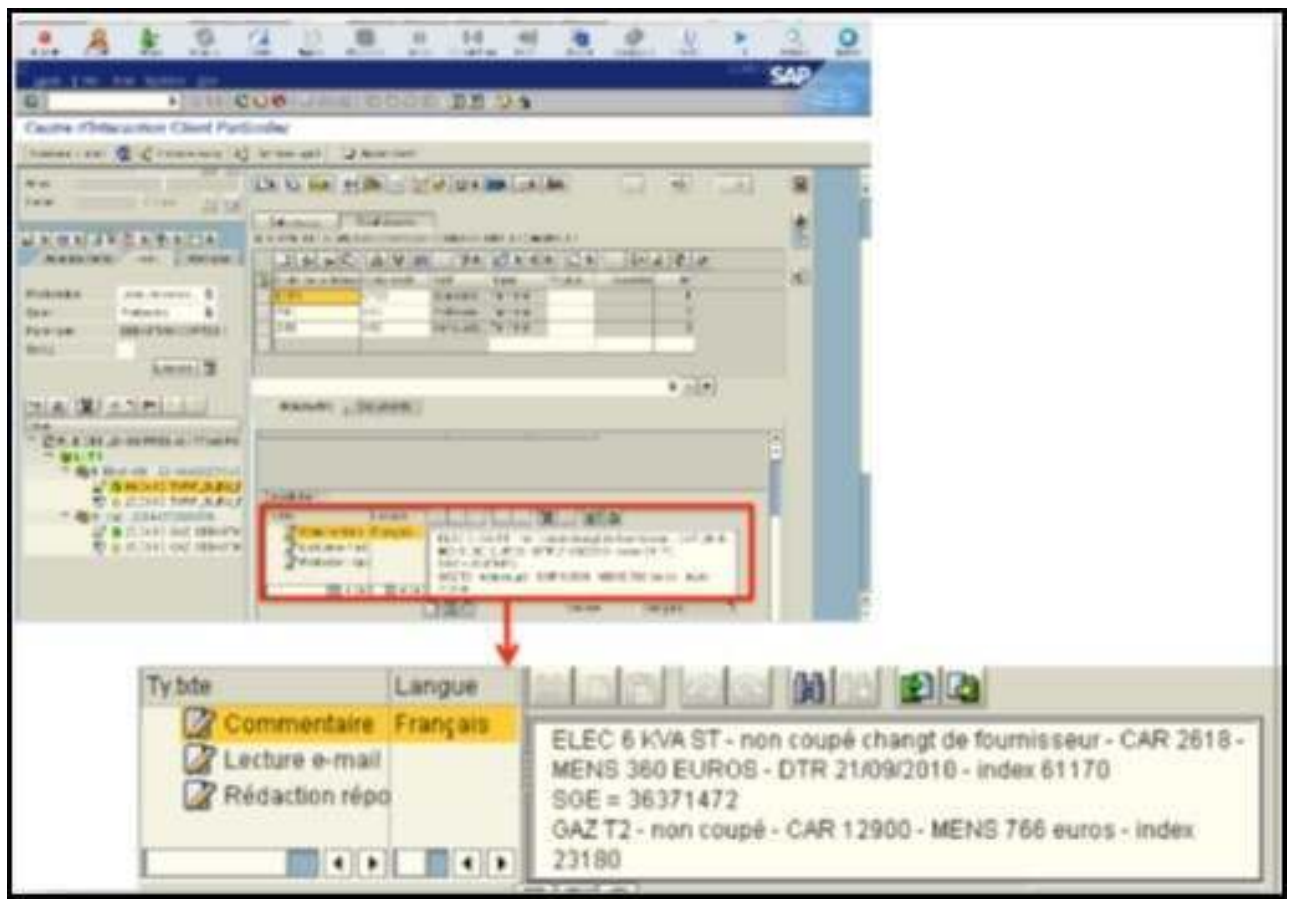

Après avoir résolu l'intrigue qui lui permet de prendre le relais et fait ce qu'il faut pour traiter l'étape actuelle qui lui incombe, le conseiller-client prépare la reprise ultérieure de ce traitement par quelqu'un d'autre. Pour cela, il dispose de capacités de transmission et de mise en archives. La distinction entre transmission et mise en archives est fondée par la connaissance dont le conseiller-client traitant l'étape actuelle dispose sur les besoins des acteurs suivants. S'il a identifié la fonction suivante dans le processus et qu'il connaît les besoins pour le traitement de cette étape ultérieure, le conseiller-client traitant l'étape actuelle transmettra directement ces éléments, car il les sait pertinents pour la suite du traitement de la demande. S'il n'a pas identifié la fonction suivante dans le processus et/ou qu'il ne connait pas les besoins relatifs à cette fonction, le conseillerclient traitant l'étape actuelle mettra en archives des éléments relatifs à sa propre activité qu'il pense pouvoir être utile à d'autres ultérieurement. Les instruments qui permettent 
de transmettre et de mettre en archives à destination des autres acteurs du traitement de la demande constituent des Capacités de transmission et de mise en archives, et articulent deux instruments :

- un instrument de partage d'éléments de son expérience située: il permet de laisser des éléments de sa propre activité que l'on sait ou pense utiles pour ceux qui interviendront ultérieurement dans le traitement de cette demande. Pour le conseiller-client, il s'agit de sélectionner, copier-coller, rédiger des commentaires à destination de ceux qui interviendront plus tard dans le traitement de la demande. Les ressources artefactuelles mobilisées dans cet instrument sont constituées par les zones du système d'information où il est possible de rédiger des commentaires libres ${ }^{8}$, ou bien par le client si ces zones ne sont pas disponibles ;

- un instrument de facilitation de la reprise efficace de la demande par autrui : il permet d'intégrer des éléments résultant de son activité ici et maintenant dans le système d'information de telle sorte que ces éléments soient immédiatement visibles et pris en compte par le prochain acteur qui interviendra sur le traitement de la demande. Pour le conseiller-client il s'agit de sélectionner, copier-coller, rédiger des commentaires, mais également de re-typer certaines zones. Les ressources artefactuelles sont constituées par différents champs du système d'information qui seront immédiatement visibles par les acteurs suivants.

Les Capacités d'enquête et les Capacités de transmission/mise en archives constituent les Capacités d'agir ensemble qui permettent aux conseillers-clients de coopérer pour traiter collectivement la demande du client. Ces Capacités d'agir ensemble sont des Instruments Transitionnels Coopératifs (Poret, 2015). Ils sont dits «transitionnels » (Bationo-Tillon, 2006; Bationo-Tillon, Folcher \& Rabardel, 2010), car ils transitent d'une étape du traitement de la demande à une autre, et "coopératifs " (Poret, 2015), car ils sont mobilisés de façon distribuée entre les différents acteurs du collectif transverse et transactionnel.

\subsection{Des instruments de la continuité temporelle au cœur des organisations}

Ces instruments transitionnels coopératifs sont au service de l'articulation Passé-PrésentFutur à chaque étape, car ils permettent à l'acteur traitant l'étape actuelle de la demande à la fois de reconstruire des dimensions passées par le moyen de la mise en intrigue, et d'anticiper le futur de ce traitement par le moyen de la transmission/mise en archives. En cela ils permettent la présentification du passé et la présentification du futur du traitement d'une demande à chaque acteur engagé dans le traitement d'une étape de cette demande. Si on quitte ce point de vue par étape pour élargir la focale et adopter le plan général du traitement de la demande, ces instruments permettent une transition entre le futur du traitement de la demande envisagée par l'acteur 1 à t, et le passé de ce traitement envisagé par l'acteur 2 à t+1 (Figure 8). En cela, ils constituent les instruments de la continuité temporelle au cœur des organisations. 
Figure 8 : Les Instruments Transitionnels Coopératifs, instruments de la continuité temporelle au cœur des organisations.

Figure 8: The Cooperative Transitional Instruments, instruments of the temporal continuity at the heart of organizations

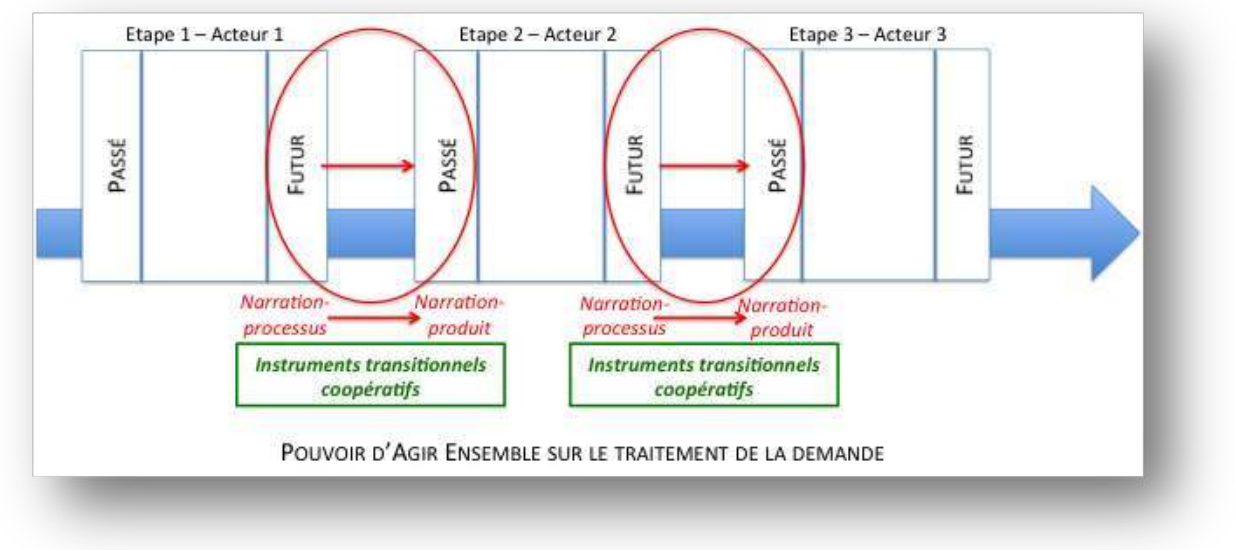

En effet, ils permettent la transition entre une étape 1 à $t$ et une étape 2 à $t+1$, en maintenant une certaine continuité. Cette continuité, à l'inverse des instruments transitionnels mis en évidence par Bationo-Tillon (2006) relativement à l'activité individuelle, ne peut pas être une continuité de l'expérience puisque l'acteur qui agit au niveau de l'étape 1 à $t$ et celui qui agit au niveau de l'étape 2 à $t+1$ ne sont pas les mêmes. Autrement dit, ces instruments permettent de maintenir une certaine continuité dans le traitement de la demande, malgré une discontinuité de l'expérience entre chacune des étapes. Cette discontinuité de l'expérience entre les différentes étapes est à l'origine du fait que les acteurs ne peuvent pas faire appel à leur mémoire personnelle. C'est la raison pour laquelle, à l'instar des historiens, ils mettent en œuvre une activité d'enquête historique dont l'objectif est de reconstruire des dimensions passées du traitement de la demande.

L'examen des ressources artefactuelles constitutives des Capacités d'agir ensemble montre que la narration y est structurelle. En effet, pour prendre le relais du traitement de la demande les conseillers-clients mettent en intrigue l'activité de ceux qui ont précédé dans ce traitement. Le matériau mobilisé et mis en intrigue est constitué par les commentaires qui ont été laissés par les acteurs qui ont précédé dans le traitement de la demande. Autrement dit, le matériau utilisé pour résoudre les intrigues est constitué par les produits de la narration des acteurs précédents dans le traitement de la demande, des «narrations-produits » (Giroux \& Marroquin, 2005). À partir de la mise en intrigue de ce matériau, les conseillers-clients aboutissent à un récit plausible de ce qui s'est passé pour le traitement de cette demande jusqu'à aujourd'hui, leur permettant de mettre en œuvre ce qu'il faut pour traiter l'étape qui leur incombe. Pour préparer la reprise ultérieure du traitement de la demande, les Conseillers Clients s'engagent à leur tour dans une "narration-processus » (Giroux \& Marroquin, ibid) où ils laissent des commentaires à destination des acteurs ultérieurs, relativement à des éléments de leur activité ici et maintenant.

Enfin, les instruments transitionnels coopératifs permettent d'enquêter et de transmettre/mettre en archives en dépassant les frontières classiques de l'organisation. Pour résoudre l'intrigue qui leur permet de prendre le relais dans le traitement de la 
demande les Conseillers Clients ont besoin d'informations et de traces laissées par les acteurs de leur entreprise, mais également de traces laissées par les acteurs du distributeur relativement au traitement de la demande en cours, afin de pouvoir intégrer ces éléments dans leur reconstruction historique, dans leur récit plausible de ce qui s'est passé avant leur contribution. Les frontières de ces instruments ne correspondent donc pas aux frontières des différents artefacts proposés, mais empruntent à différents artefacts relatifs à différentes entités engagées dans le processus.

\section{Concevoir pour le pouvoir d'agir ensemble : le critère de continuité pour la conception technico-organisationnelle}

\subsection{Présentation du critère de continuité}

43 À partir de la compréhension des fondements du Pouvoir d'agir ensemble et pour ancrer durablement la transversalité au sein des organisations, nous proposons un critère pour la conception technico-organisationnelle. L'efficacité pratique qui est recherchée avec la mise en place de ce critère étant constituée par une exigence de continuité du traitement de la demande à partir d'une continuité de l'activité collective transverse et transactionnelle, nous avons nommé ce critère un « critère de continuité » (Poret, 2015). En proposant ce critère généralisable de conception, nous souhaitons dépasser le stade d'ajustements locaux spécifiques à un environnement technico-organisationnel donné pour impulser un mouvement de transformation durable des dispositifs technicoorganisationnels selon une logique transverse.

La première exigence pour la mise en œuvre de ce critère consiste à identifier l'ensemble des acteurs et l'ensemble des étapes prévues et aléas qui doivent s'articuler pour aboutir à l'objectif final du processus : la production d'un service ou d'un bien à destination d'un client. Cette identification doit intégrer les acteurs et les étapes à la fois de manière intraet inter- organisationnelle et se faire depuis un point d'entrée par l'activité individuelle, réelle et située des acteurs du collectif transverse et transactionnel. Il ne s'agit pas de procéder à un recensement des étapes officielles constitutives d'un processus, mais de s'immerger dans le processus à n'importe quelle étape, afin de reconstruire ce dernier à partir des éléments de l'activité individuelle et collective, réelle et située de l'acteur chargé de traiter cette étape. Dans nos analyses nous avons été confrontés à des configurations où le Pouvoir d'agir ensemble des acteurs est impacté parce que cette identification n'a pas été réalisée. C'est par exemple le cas lorsque le client souhaite avoir des informations sur le traitement de sa demande qui est en train d'être réalisé par un prestataire avec lequel le conseiller-client ne dispose d'aucun espace commun dans le système d'information. Il lui est alors très difficile de récupérer le matériau nécessaire à la résolution de l'intrigue historique caractéristique de la prise de relais dans une activité collective transverse et transactionnelle. Cela complexifie son travail et peut également impacter négativement la satisfaction du client.

Coopérer de façon transverse à l'organisation consiste à articuler Passé-Présent-Futur à chaque étape. Les instruments transitionnels coopératifs au service de cette activité collective permettent justement de présentifier le passé et le futur dans le cadre de 
l'étape actuelle. Les deux autres exigences de la mise en œuvre du critère de continuité portent sur ces aspects.

- la seconde exigence consiste en effet à concevoir pour la présentification du passé, pour la mémoire. Prendre la suite dans une activité collective transverse et transactionnelle consiste à résoudre des intrigues historiques, différentes selon l'étape de cette activité collective dans laquelle on intervient. Concevoir pour le Pouvoir d'agir ensemble consiste à aider à la résolution de ces intrigues en rendant présents des éléments issus du passé, voire un décours temporel d'évènements jusqu'à l'instant $t$ afin que l'acteur engagé dans le traitement de l'étape actuelle sache ce qu'il faut effectivement mettre en œuvre pour prendre le relais. Il s'agit de proposer des supports de la mémoire pour que la prise de relais soit possible malgré le fait que les étapes antérieures n'aient pas été traitées par l'acteur qui est engagé dans l'étape actuelle. Ces supports de la mémoire collective doivent permettre de s'engager efficacement dans la résolution d'une intrigue, en supportant l'activité d'enquête historique mise en œuvre pour cela par les acteurs du collectif transverse et transactionnel. L'objectif de cette présentification du passé est de rendre immédiatement visible une histoire : celle des différentes contributions ayant mené à l'état du processus à l'instant $t$, à partir de laquelle il faut construire sa propre contribution. Cette présentification du passé est également utile pour le client qui a besoin de «suivre et maîtriser l'évolution de sa demande, mieux comprendre l'organisation des métiers de l'entreprise [et] rendre plus efficace son implication dans le processus de traitement de sa demande » (Motté \& Haradji, 2010, p. 27).

- la troisième exigence consiste à concevoir pour la présentification du futur, pour l'attente. Préparer la suite dans une activité collective transverse et transactionnelle consiste à transmettre et à mettre en archives des éléments de sa propre activité à destination des acteurs qui auront à intervenir ultérieurement dans l'activité collective transverse et transactionnelle. Concevoir pour le Pouvoir d'agir ensemble consiste à rendre possible cette transmission et cette mise en archives d'éléments non directement pertinents pour soi à l'instant $t$, mais pour de futurs acteurs plus tard et ailleurs. Il s'agit d'anticiper ici et maintenant le futur de l'activité collective transverse et transactionnelle, c'est-à-dire d'anticiper les attentes des acteurs qui interviendront plus tard et ailleurs dans cette activité collective. La proposition principale que nous faisons pour permettre la présentification du futur consiste à penser l'intégration de tous les acteurs du collectif transverse et transactionnel au sein d'espaces communs dans le système d'information. La confrontation de pratiques évoquée dans la seconde partie de cet article joue ici un rôle crucial, car une transmission et un adressage efficaces d'éléments de son activité à destination de ceux qui interviendront ultérieurement dans le traitement de la demande impliquent de connaître leurs besoins, et donc d'avoir conscience des interdépendances entre sa propre contribution à l'instant $t$ et celles de ces futurs acteurs dans le cadre d'une activité collective transverse et transactionnelle.

\subsection{La conception d'une aide à la continuité du traitement de la demande : le graphe interactif de suivi du traitement de la demande}

46 Ce critère a été mobilisé au sein de deux projets opérationnels de chez Électricité de France (EDF) : un projet de conception d'un graphe interactif de suivi du traitement de la demande, prévu pour s'afficher à l'ouverture du dossier d'un client par le conseiller-client qui pourra alors immédiatement voir l'état d'avancement du traitement de la demande, ainsi qu'un projet de cartographie d'un processus de l'organisation depuis une 
connaissance de l'activité collective transverse et transactionnelle. Nous présentons plus en détail la façon dont le critère de continuité a permis de structurer le premier projet.

Le graphe interactif de suivi du traitement de la demande est une innovation proposée par les chercheurs en ergonomie de la Direction Recherche \& Développement d'Électricité de France (EDF) dans le cadre de leur programme de recherche «Ingénierie pour la relation de service ", en vue d'aider l'activité collective transverse et transactionnelle. À partir d'une modélisation de l'activité collective transverse et transactionnelle, et en collaboration avec des informaticiens, nous avons été amenés à réfléchir aux principes de conception et à concevoir une maquette de ce graphe. Ce dernier a plusieurs caractéristiques (Motté, 2012) :

- il rend visible la construction collective du traitement d'une demande par chaque acteur contributeur (différents acteurs EDF, client, acteurs du distributeur) ;

- il s'appuie sur les outils SI existants et sur la remontée des éléments significatifs issus du traitement effectué dans ces outils. Il est donc alimenté sans qu'aucune activité humaine ne soit nécessaire, ce qui permet de ne pas alourdir l'activité de chacun des acteurs engagés dans l'activité collective transverse et transactionnelle ;

- il propose en parallèle une vue côté EDF et une vue côté client permettant ainsi à chacun de suivre l'avancement de la demande et d'agir en conséquence. Le graphe permet donc d'outiller à la fois la dimension "transverse " de l'activité collective qui est relative à la coopération des acteurs professionnels pour construire collectivement le service au client, mais également la dimension « transactionnelle » de cette activité collective qui est relative au fait que cette dernière implique le client avec lequel l'objectif est de parvenir à une «transaction » : la production de son service;

- il permet un accès rapide et direct aux applications métier. Le graphe ne constitue pas qu'une représentation partagée de la demande et de son avancement, mais également un point d'entrée dans les outils existants.

En rendant visible une chronologie d'évènements intervenus dans le traitement de la demande qui construisent progressivement ce traitement, il rend possible une présentification du passé à chaque étape, exigence requise par la mise en œuvre du critère de continuité. Ce graphe constitue une aide à l'activité d'enquête historique qui caractérise la prise de relais dans le traitement transverse et transactionnel d'une demande. Le conseiller-client qui ouvrirait le dossier d'un client pourrait alors immédiatement l'état du traitement de la demande en cours, ainsi qu'un décours temporel d'évènements expliquant cet état. Selon l'étape dans laquelle s'engage le conseiller-client, ce décours temporel ne focaliserait pas sur les mêmes dimensions. L'activité d'enquête historique concerne des intrigues différentes selon l'étape dans laquelle est engagé le conseiller-client. Les informations considérées pour la résolution de ces intrigues ne sont donc pas les mêmes à chaque étape, et vont se focaliser sur des dimensions différentes. Par exemple, l'intrigue dans laquelle s'engage un conseiller-client chargé de traiter une étape de traitement du contrat gaz signé est « La contractualisation est-elle toujours possible?», et les informations sélectionnées pour y répondre se focalisent sur la contractualisation chez Électricité de France (EDF) et chez le distributeur. Aussi, l'intrigue dans laquelle s'engage un conseiller-client qui doit traiter une étape d'anomalie n'est pas la même, puisqu'il s'agit de « Qu'est-ce qui a mené cette demande en anomalie? », et les informations sélectionnées pour y répondre se focalisent donc sur l'histoire de la demande chez Électricité de France (EDF) et chez le distributeur. L'histoire à reconstruire pour prendre le relais dans une activité collective transverse et 
transactionnelle n'est donc pas la même selon les étapes dans lesquelles les conseillersclient interviennent. Nous avons proposé des modes d'affichage différents du graphe selon l'étape et donc l'intrigue concernées.

Figure 9 : Logique du graphe pour la résolution de l'intrigue « La contractualisation est-elle toujours possible?"

Figure 9: Logic of the graph to unravel the "Is contractualization always possible?"

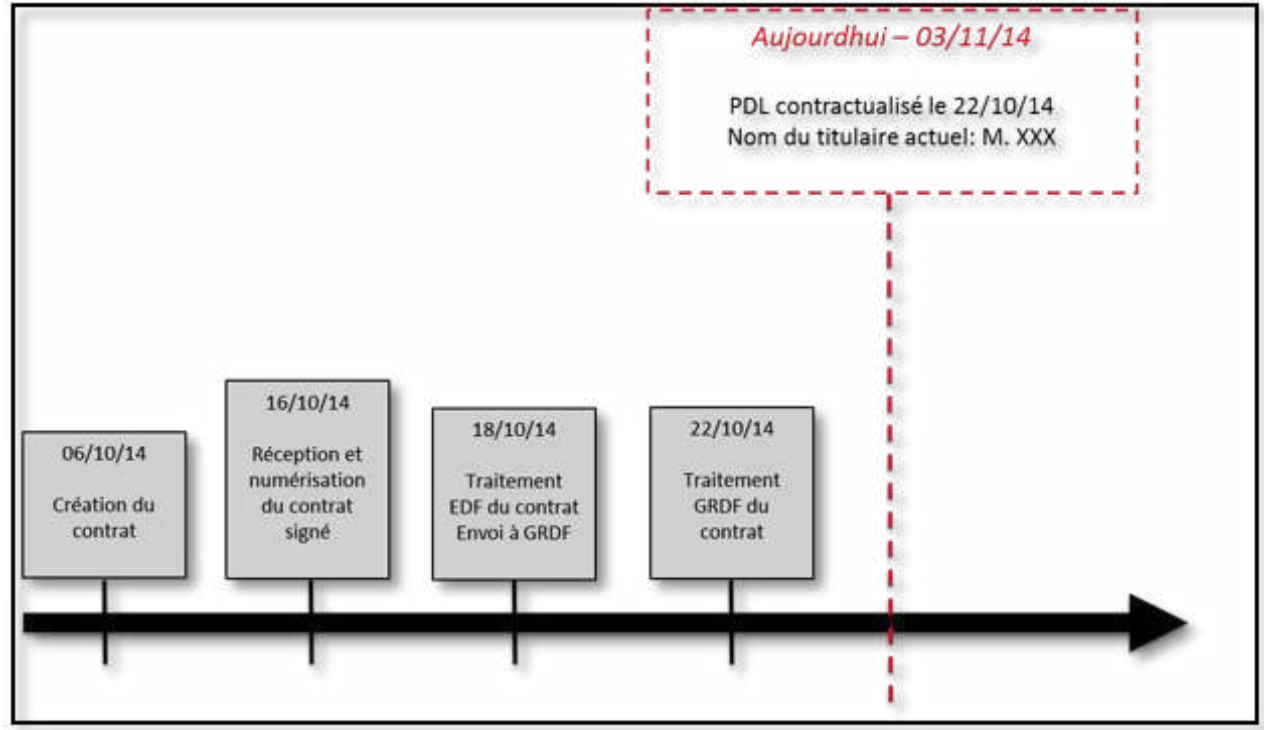

La figure 9 illustre par exemple la logique de ce graphe pour un conseiller-client engagé dans une étape de traitement du contrat gaz signé. Ce graphe affiche des éléments relatifs à l'histoire de la contractualisation pour un logement, chez Electricité de France (EDF) et chez le distributeur, en vue de résoudre l'intrigue « La contractualisation est-elle toujours possible?». Nous exemplifions ici une façon de présentifier le passé pour outiller la dimension «transverse» de l'activité collective transverse et transactionnelle. Cette présentification $\mathrm{du}$ passé est également prévue pour outiller sa dimension « transactionnelle » en permettant au client de disposer d'un tel graphe - moyennant un affichage d'informations différentes - afin de lui rendre visible l'état d'avancement du traitement de sa demande. Ce graphe pourrait être affiché dans son espace-client et participerait ainsi à un renforcement de la Gestion de la Relation Client (GRC) via internet.

\section{Conclusion et discussion}

Cette recherche s'est inscrite dans un contexte de transformation des organisations et des métiers de la relation de service chez Electricité de France (EDF), suite à l'ouverture du marché de l'énergie à la concurrence. Il s'agissait de comprendre la dynamique de l'activité collective transverse et transactionnelle entre les acteurs d'un même processus qui doivent coopérer pour parvenir à l'objectif final de ce processus : la production du service au client. Des auteurs issus du monde l'ergonomie et de la gestion ont mis en évidence l'importance de cette activité collective et proposé des dispositifs de confrontation de pratiques pour l'outiller. Or, cette façon de concevoir pour l'activité collective transverse et transactionnelle est limitée, car elle se centre uniquement sur les 
acteurs engagés dans cette activité, laissant de côté l'environnement technique et organisationnel dans lequel elle doit pouvoir se déployer. Aussi, si la conception pour ce type d'activité collective ne considère pas le rapport « Acteur-Environnement » le risque est de ne permettre que la construction de connaissances indispensables pour coopérer sans toutefois pouvoir les mobiliser en situation. Le risque est alors de permettre la seule construction de Capacités d'agir ensemble sans se poser systématiquement la question de leur mobilisation effective en situation: le Pouvoir d'agir ensemble (Poret, 2015). Nous avons montré et décrit les ressources de ce Pouvoir d'agir ensemble au cœur d'une activité collective transverse et transactionnelle: les instruments transitionnels coopératifs (Poret, ibid), qui permettent d'articuler Passé-Présent-Futur à chaque étape et ainsi de coopérer au sein d'une activité collective distribuée temporellement, spatialement, entre plusieurs acteurs. Enfin, nous avons proposé un critère pour la conception de systèmes technico-organisationnels qui ancrent durablement la transversalité au cœur des organisations en permettant le Pouvoir d'agir ensemble - le critère de continuité - et avons illustré sa mobilisation dans le cadre d'un projet opérationnel de chez Electricité de France (EDF).

S'engager dans un travail de recherche implique de faire un pari sur la nature des choses (Theureau, 2002a, 2002b). Aussi, pour appréhender les fondements du Pouvoir d'agir ensemble, nous avons choisi d'adopter une maille d'analyse individuelle-sociale. Ce choix est fondé par des considérations ontologiques, épistémologiques et méthodologiques qui considèrent structurellement l'asymétrie $\mathrm{du}$ rapport entre l'Homme et son environnement et accordent donc le primat au point de vue de l'acteur pour la connaissance de son activité. Cette maille d'analyse n'est pas unanimement partagée par l'ensemble des auteurs qui s'intéressent à l'activité collective transverse et transactionnelle. Par exemple, Lorino $(2009,2013)$ adopte une maille d'analyse dialogique, d'emblée collective, sans passer par la maille d'analyse individuelle-sociale. Nous argumentons la pertinence et la force de la maille d'analyse individuelle-sociale pour l'étude de l'activité collective transverse et transactionnelle pour différentes raisons. Tout d'abord, parce que seule la maille d'analyse individuelle-sociale permet d'accéder aux instruments subjectifs construits par les acteurs au service de leur activité et donc de s'engager dans la conception en dépassant la seule centration sur les acteurs. Nous argumentons que seule cette maille d'analyse individuelle-sociale permet de s'engager dans la conception technique, essentielle pour l'existence de l'activité collective transverse et transactionnelle au sein des organisations. De plus, la maille d'analyse individuelle-sociale permet d'accéder à des phénomènes d'adressage de l'activité, comme cette recherche en témoigne. En effet, coopérer dans le cadre d'une activité collective transverse et transactionnelle consiste - à chaque étape de ce traitement - à mettre en intrigue des éléments qui ont été adressés par d'autres et à adresser à son tour des éléments pour de futures mises en intrigue par les acteurs qui interviendront ultérieurement. Accorder le primat au point de vue de l'acteur correspond ainsi à une voie qui permet de réintégrer l'acteur isolé par une ontologie subjectiviste et exclu par une ontologie holiste.

Nous pensons que ces deux mailles d'analyse - dialogique et individuelle-sociale - sont complémentaires pour la compréhension de l'activité humaine, puisqu'elles ont notamment permis la mise en évidence de deux types d'enquête interdépendants qui interviennent au cœur des organisations: 1) une enquête inter-métiers qui crée les conditions préalables à la coopération transverse et transactionnelle puisqu'il s'agit 
d'enquêter sur les besoins des autres acteurs pour les intégrer dans sa propre activité et 2) une enquête historique qui permet de prendre le relais et de coopérer dans le cadre d'une activité collective transverse et transactionnelle. Elles sont également complémentaires pour la conception puisque l'une propose de concevoir pour construire les conditions préalables à l'activité collective et l'autre de concevoir pour permettre son existence en situation, c'est-à-dire dans un environnement technico-organisationnel qui fragmente cette activité. Pour ces différentes raisons, nous pensons que c'est l'articulation entre ces deux mailles d'analyse et entre les apports complémentaires de l'une et de l'autre en termes de connaissances de l'activité humaine et de conception qui permettra d'ancrer durablement la transversalité au cœur des organisations.

Eu égard à cette nécessité d'ancrer la transversalité au cœur des organisations, certains auteurs (Hammer, 1990; Hammer \& Champy, 1993) proposent la réingénierie des processus (Business Process Reengineering) pour améliorer la performance de l'entreprise. L'un des principes de cette réingénierie (Hammer, 1990) consiste à rendre les agents polyvalents, c'est-à-dire à faire en sorte qu'un seul agent traite l'ensemble des étapes du processus. On pourrait alors penser que le traitement d'une demande en différentes étapes est un découpage artificiel qui complexifie ce traitement et que la polyvalence est la solution pour ancrer la transversalité dans les organisations. La portée de la présente recherche serait alors quasi-nulle, et le critère de continuité inutile puisqu'il suffirait d'instaurer la polyvalence des agents pour que cette continuité soit effective. La nécessité de permettre une continuité de l'activité collective entre plusieurs personnes ne serait plus d'actualité puisqu'on y aurait substitué la continuité de l'expérience pour une seule et même personne! Les choses ne sont pourtant pas aussi simples. Tout d'abord, le fait que le traitement d'une demande de mise en service bi-énergie implique des métiers différents - du distributeur et du commercialisateur - entraîne de fait des étapes différentes. Les spécificités liées à des différences de métiers constituent donc une limite à ce principe de réingénierie de processus; le métier du conseiller-client ne consiste pas à réaliser l'intervention de raccordement au domicile du client effectuée par le distributeur. De plus, le marché de l'énergie est caractérisé par un grand nombre de consommateurs; par exemple, EDF compte 25 millions de clients Particuliers en France 9 Même si la polyvalence était rendue possible pour l'ensemble des étapes du traitement de la demande de mise en service bi-énergie, tous métiers confondus, cette dimension mass market rend impossible le suivi individualisé par un seul conseiller-client d'une seule et même demande client. Ces deux caractéristiques - métiers différents et mass market sont à l'origine du fait que la polyvalence des agents ne peut constituer une solution unique pour permettre la continuité du traitement de la demande du client et donc la performance de l'entreprise pour répondre à ces demandes. D'ailleurs, les conseillersclients EDF sont polyvalents. En étant engagés à la fois dans des activités de front et de back-office, ils sont amenés à traiter différentes étapes d'un même processus. Par exemple, dans le traitement de la demande de mise en service bi-énergie, les conseillersclients peuvent traiter l'étape de souscription (étape $n^{\circ} 1$ ) ou l'étape de traitement du contrat gaz signé (étape $\mathrm{n}^{\circ} 2$ ), voire d'autres étapes supplémentaires qui peuvent résulter de traitements différés ou d'anomalies. Du coup, différentes étapes d'un même processus leur ont été attribuées, à l'instar de ce qui est proposé par la réingénierie des processus. Alors que le traitement de ces différentes étapes du processus leur incombe, il ne leur est pourtant pas possible de traiter ces étapes pour une seule et même demande concernant un seul et même client. Polyvalence n'est donc pas synonyme de suivi personnalisé du 
traitement d'une demande client pour un seul et même client; elle ne constitue donc pas la clé de la continuité et de la transversalité dans les organisations.

Enfin, cette exigence d'ancrage de la transversalité dans les organisations va très certainement s'accentuer dans les années à venir. D'une manière générale, les configurations organisationnelles qui impliquent le développement d'une activité collective transverse et transactionnelle vont continuer de se développer, dans des secteurs divers et variés. C'est par exemple le cas dans le domaine médical où la nécessité de favoriser la continuité des soins (continuity of care) entre des prestataires de différents métiers va très certainement être de plus en plus importante au vu du contexte économique et de l'accroissement des hospitalisations à domicile. Dans le domaine énergétique, le déploiement des technologies de l'information et de la communication (TIC) sur les réseaux publics d'électricité et de gaz va permettre - moyennant la gestion des données remontées par de telles technologies - de proposer des services personnalisés et adaptés au client. Ce « sur-mesure de masse » (Roy \& Audet, 2002) risque de complexifier les processus actuels, notamment du fait du nombre croissant d'acteurs qui seront intégrés pour fournir un tel service.

\section{BIBLIOGRAPHY}

Arnoud, J. (2013). Conception organisationnelle : pour des interventions capacitantes. Thèse de doctorat, Conservatoire National des Arts et Métiers, Paris.

Arnoud, J., \& Falzon, P. (2013). La co-analyse constructive des pratiques. In P. Falzon (Ed.), Ergonomie constructive (pp. 223-236). Paris : Presses Universitaires de France.

Arnoud, J., \& Falzon, P. (2014). Favoriser l'émergence d'un collectif transverse par la co-analyse constructive des pratiques. Le Travail Humain, 77 (2), 127-153.

Askenazy, P. (2005). Charge mentale au travail : les contributions d'une approche économique. In J. Furtos, \& C. Laval (Eds.), La santé mentale en actes (pp. 127-151). ERES, Études, recherches, actions en santé mentale en Europe.

Bationo Tillon, A. (2006). Pratiques des activités narratives instrumentées : Une analyse diachronique et structuro-fonctionnelle en amont de la conception. Université Paris $8:$ Thèse de Doctorat en Ergonomie.

Bationo, A., Folcher, V., \& Rabardel, P. (2010). Les instruments transitionnels une proposition pour étudier la diachronie des activités narratives. Activités, 7(2), 63-83. http:// activites.revues.org/2437.

Bernard, S. (2005). Le temps de l'activité de la caissière entre logique productive et logique de service. Sociologie du travail, 47, 170-187.

Bidet, A., Boutet, M., \& Chave, F. (2013). Au-delà de l'intelligibilité mutuelle : l'activité collective comme transaction. Un apport du pragmatisme illustré par trois cas. Activités, 10(1), 172-191. http://activites.revues.org/632.

Boreham, N., Samurçay, R., \& Fischer, M. (2002). Work Process Knowledge. London : Routledge. 
Buscatto, M. (2002). Les centres d'appels, usines modernes? Les rationalisations paradoxales de la relation téléphonique. Sociologie du travail, 44, 99-117.

Calderon, J. (2006). Le travail face à la restructuration productive : le cas d'un centre d'appels. Formation emploi, 96, 11-24.

Cerf, M., Valléry, G., \& Boucheix, J.-M. (2004). Les activités de service : enjeux et développements. In P. Falzon (Ed.), Ergonomie (pp. 565-581). Paris : Presses Universitaires de France.

Cihuelo, J. (2010). Le cas d'un modèle hybride de centre d'appels. Entre rationalisation et réappropriation professionnelle. Réseaux, 164, 164-196.

Daniellou, F., \& Rabardel, P. (2005). Activity-oriented approaches to ergonomics: some traditions and communities. Theoretical Issues in Ergonomics Science, 6(5), 353-357.

Defelix, C. (1999). Transversalité, contrôle et gestion des ressources humaines : un cas de pilotage à l'épreuve des processus. Finance Contrôle Stratégie, 2(2), 51-70.

Derujinsky-Laguecir, A., Kern, A., \& Lorino, P. (2011). Une approche instrumentale des indicateurs de performance. Management \& Avenir, 2(42), 111-132.

Detchessahar, M. (2003). L'avènement de l'entreprise communicationnelle. Revue française de gestion, 1(142), 65-84.

Dewey, J., \& Bentley, A. F. (1949). Knowing and the Known. Boston : The Beacon Press.

Du Tertre, C. (2013). Économie servicielle et travail : contribution théorique au développement « d'une économie de la coopération ». Travailler, 1(29), 29-64.

Flageul-Caroly, S. (2001). Régulations individuelles et collectives des situations critiques dans un secteur des services : le guichet de La Poste. Paris : Thèse, LEPC-EPHE.

Folcher, V., \& Rabardel, P. (2004a). Hommes, artefacts, activités : perspective instrumentale. In P. Falzon (Ed.), Ergonomie (pp. 251-268). Paris : Presses Universitaires de France.

Folcher, V., \& Rabardel, P. (2004b). Artifacts as design-for-use propositions for design-in-use activity. Invited conference to the symposium "Perception, Communication and Activity". International Congress of Psychology (ICP 2004), 7-13 Août 2004, China.

Giroux, N., \& Marroquin, L. (2005). L'approche narrative des organisations. Revue Française de Gestion, 6(159), 15-44.

Greenan, N., \& Mairesse, J. (2006). Les changements organisationnels, l'informatisation des entreprises et le travail des salariés. Un exercice de mesure à partir de données couplées entreprises/salariés. Revue économique, 57(6), 1137-1175.

Hammer, M. (1990). Reengineering Work: don't automate, obliterate. Harvard Business Review, 104-114.

Hammer, M., \& Champy, J. (1993). Le Reengineering (édition française). Paris : DUNOD.

Hochereau, F. (2004). L'évolution de la fonction commerciale à France Telecom (1978-1998) à travers son informatisation. In L. Deroche, \& G. Jeannot (Eds.), L'action publique au travail (pp. 17-24). Toulouse : Octarès Éditions.

Khoshafian, S., \& Buckiewicz, M. (1998). Groupware \& workflow. Paris : Masson.

Lasnier, G. (2007). Le lean-manufacturing (système de production à haute performance) dans les industries travaillant en juste-à-temps avec flux régulés par takt-time (rythme de la consommation du client). La Revue des Sciences de Gestion, 1(223), 99-107. 
Lave, J., \& Wenger, E. (1991). Situated learning. Legitimate peripheral participation. Cambridge : Cambridge University Press.

Lorino, P. (2009). Concevoir l'activité collective conjointe : l'enquête dialogique. Étude de cas sur la sécurité dans l'industrie du bâtiment. Activités, 6(1), 87-110. http://activites.revues.org/2154. Lorino, P. (2013). L'activité collective, processus organisant. Un processus discursif fondé sur le langage pragmatiste des habitudes. Activités, 10(1), 1-24. http://activites.revues.org/656.

Lorino, P., \& Peyrolle, J.-C. (2005). Démarche pragmatiste et mise en processus dans les situations de gestion. In R. Teulier, \& P. Lorino (Eds.), Entre connaissance et organisation : l'activité collective (pp. 220-229). Paris: La Découverte.

Lorino, P., \& Tricard, B. (2012). The Bakhtinian Theory of Chronotope (Time-Space Frame) Applied to the Organizing Process. In M. Schultz, S. Maguire, A. Langley, \& H. Tsoukas (Eds.), Constructing identity in and around Organizations (pp. 201-234). Oxford: Oxford University Press.

Maturana, H., \& Varela, F. (1980). Autopoiesis and Cognition. Dordrecht : Reidel.

Maturana, H., \& Varela, F. (1987). The tree of knowledge. Boston/London : Shambala.

Motté, F. (2012). Le collectif transverse : un nouveau concept pour transformer l'activité. In Actes du 47ème Congrès International de la Société d'Ergonomie de Langue Française (SELF) (pp. 102-108).

Motté, F., \& Haradji, Y. (2010). Construire la relation de service en considérant l'activité humaine dans ses dimensions individuelles et collectives. In G. Vallery, M.-C. Le Port, \& M. Zouinar (Eds.), Ergonomie et conception de produit et de services médiatisés (pp. 11-35). Paris : Presses Universitaires de France.

Mourlon, S., \& Neyer, L. (2002). Tout ce que nous avons voulu savoir sur les ERP. Qu'attendre des Progiciels de Gestion Intégrés? Mémoire d'Ingénieurs Elèves - École des Mines de Paris.

Nascimento, A. (2009). Produire la santé, produire la sécurité. Développer une culture collective de sécurité en radiothérapie. Paris : Thèse de doctorat du Conservatoire National des Arts et Métiers (CNAM).

Petit, J. (2005). Organiser la continuité du service : Intervention sur l'organisation d'une Mutuelle de santé. Thèse pour l'obtention du grade de docteur en ergonomie de l'université Bordeaux 2.

Poret, C. (2015). Concevoir pour le Pouvoir d'Agir Ensemble d'un collectif transverse. Le cas de la relation de service dans le domaine commercial. Thèse de doctorat - Université Paris VIII.

Rabardel, P. (1995). Les Hommes et les technologies. Paris : Armand Colin.

Rabardel, P. (2005a). Instrument subjectif et développement du pouvoir d'agir. In P. Rabardel \& P. Pastré (Eds.), Modèles du sujet pour la conception. Dialectiques activités développement. Toulouse : Octarès Éditions.

Rabardel, P. (2005b). Instrument, activité et développement du pouvoir d'agir. In R. Teulier, \& P. Lorino (Eds.), Entre connaissance et organisation : l'activité collective (pp. 251-265). Paris: La Découverte.

Rabardel, P., \& Béguin, P. (2005). Instrument mediated activity: from subject development to anthropocentric design. Theoretical Issues in Ergonomics Science, 6(5), 429-461.

Rabardel, P., Folcher, V., \& Béguin, P. (2002). Les instruments pour l'action : une approche développementale. Communication à l'atelier de conjoncture de la Société Française de Psychologie (SFP), Octobre 2002, CNAM-Paris. 
Roy, M., \& Audet, M. (2002). La transformation vers de nouvelles formes d'organisation plus flexibles : un cadre de référence. Gestion, 27(4), 43-49.

Sartre, J.-P. (1936/1972). La transcendance de l'ego. Essai d'une description phénoménologique. Paris : Vrin.

Sartre, J.-P. (1943). L'Être et le néant. Essai d'ontologie phénoménologique. Paris : Éditions Gallimard.

Segrestin, D. (2004). Les chantiers du manager. L'innovation en entreprise : où en sommes-nous? Comment piloter les changements et les maîtriser ? Paris : Armand Colin.

Sève, C., Theureau, J., Saury, J., \& Haradji, Y. (2012). Drôles d'endroits pour une rencontre : STAPS, Ergonomie et Cours d'Action. In M. Quidu (Ed.), Les sciences du sport en mouvement. Innovations et traditions théoriques en STAPS. Paris : L'Harmattan.

Tarondeau, J.-C. (2002). Le management des savoirs. Paris : PUF.

Theureau, J. (2002a). Cours d'expérience, cours d'action, cours d'interaction : essai de précision des objets théoriques de l'activité individuelle-sociale. 4e Journées Act'Ing "Objets théoriques, objets de conception, objets d'analyse et situations d'étude privilégiées", 6-7 juin, Nouan le Fuzelier.

Theureau, J. (2002b). Articulation collective des cours d'action : de l'inspiration Sartrienne à un programme de recherche. $4^{\mathrm{e}}$ Journées Act'Ing “Objets théoriques, objets de conception, objets d'analyse et situations d'étude privilégiées", 6-7 juin, Nouan le Fuzelier.

Theureau, J. (2004). Le cours d'action : méthode élémentaire. Toulouse : Octarès Éditions.

Theureau, J. (2006). Le cours d'action : méthode développée. Toulouse : Octarès Éditions.

Theureau, J. (2009). Le cours d'action : méthode réfléchie. Toulouse : Octarès Éditions.

Theureau, J. (2015). Le cours d'action. L'enaction \& l'expérience. Toulouse : Octarès Éditions.

Theureau, J., \& Jeffroy, F. (1994). Ergonomie des situations informatisées. La conception centrée sur le cours d'action de l'utilisateur. Toulouse : Octarès Éditions.

Trompette, P., \& Boissin, O. (2000). Entre les vivants et les morts : les pompes funèbres aux portes du marché. Sociologie du travail, 42, 483-504.

Ughetto, P. (2012). Le lean : pensée et impensé d'une activité sans relâchement. Activités, 9(2), 148-167. http://activites.revues.org/453

Veltz, P., \& Zarifian, P. (1994). Travail collectif et modèles d'organisation de la production. Le Travail Humain, 57(3), 239-249.

Veyne, P. (1971). Comment on écrit l'histoire. Paris : Éditions du Seuil.

Watiez, J.-C. (2002). Flexibilité et adaptation de l'organisation à l'environnement : la flexibilité en tant que réponse à l'asymétrie inter et intra entreprises. Finance Contrôle Stratégie, 5(1), 217-254.

Zarifian, P. (1995). Travail et communication. Paris : L'Harmattan.

\section{NOTES}

1. Nous entendons ici le terme "transactionnel» dans un sens courant, afin de signifier que l'activité collective dont il est question doit aboutir à une transaction créatrice de valeur avec le client. Nous ne l'entendons pas dans un sens pragmatiste tel que défini par Dewey et Bentley (1949) et repris plus récemment par Bidet, Boutet et Chave (2013). 
2. Notre traduction

3. Caractéristiques datant de l'époque de notre recueil de données ( $1^{\mathrm{er}}$ semestre 2010)

4. Au niveau national, 3,2 millions de demandes de mises en service sont traitées chaque année, d'où l'importance de s'intéresser à l'activité collective transverse et transactionnelle qui doit s'y déployer pour assurer un service de qualité à destination du client.

5. Il s'agit plus précisément d'une modélisation de l'histoire des transformations du système des ouverts (Theureau, 2006).

6. Pour la description complète de ces séquences (Theureau, 2004, 2006, 2009) à partir des Unités Significatives Elémentaires (USE) qui les constituent, voir Poret (2015)

7. Pour la caractérisation plus complète de ces instruments, entités mixtes composées de schèmes et d'artefacts, voir Poret (2015)

8. La figure 7 illustre une telle zone. La différence entre sa mobilisation dans le cadre des instruments de synthèse historique / de recherche d'une information historique ET dans le cadre de cet instrument de partage d'éléments de son expérience située est constituée par le fait que pour les instruments relatifs à l'histoire la ressource est le commentaire en tant que tel, tandis qu'elle est la zone vierge de tout commentaire pour l'instrument relatif au futur.

9. Communiqué de presse du 10 février 2016 «EDF, numéro 1 de la relation client dans la catégorie "service" "

\section{ABSTRACTS}

In a context of changes to the economic environment and structural changes in companies which must now consider their performance in terms of their cross-functional processes, it is essential to understand the grounds of an organization's efficiency when coping with its customers' demands. One of these grounds is a cross-functional cooperation between actors involved in the same process, within the framework of a new kind of collective activity: cross-functional and transactional collective activity (Motté \& Haradji, 2010 ; Poret, 2015). This collective activity requires actors who are involved in the same process to cooperate by crossing the organization's boundaries. From an understanding of the different methods of coordination which form the basis of cooperation between actors of the same cross-functional process, this research highlights the existence of instruments enabling said actors to cross the organization's classic boundaries in order to cooperate (Cooperative Transitional Instruments (Poret, ibid)) and proposes a criterion for the design of IT and organizational systems in order to durably root transversality and implement the Power to Act Together at the heart of organizations.

Dans un contexte de transformations de l'environnement économique et de changements structurels des entreprises qui doivent penser leur performance à partir de leurs processus transversaux, il est essentiel de comprendre les ressorts de l'efficacité des organisations pour répondre aux demandes de leurs clients. Un de ces ressorts est constitué par une coopération transverse entre les acteurs engagés dans un même processus, dans le cadre d'une activité collective d'un type nouveau: l'activité collective transverse et transactionnelle (Motté \& Haradji, 2010 ; Poret, 2015). Cette activité collective implique pour les acteurs du processus de coopérer de façon transverse à l'organisation. À partir de la compréhension des modes de coordination qui fondent la coopération des acteurs d'un processus transverse, cette recherche met en évidence l'existence d'instruments qui permettent à ces acteurs de dépasser les frontières 
classiques de l'organisation pour coopérer: les Instruments Transitionnels Coopératifs (Poret, ibid), et propose un critère pour la conception des systèmes technico-organisationnels afin d'ancrer durablement la transversalité et de permettre le Pouvoir d'Agir Ensemble au cœur des organisations.

INDEX

Mots-clés: relation de service, activité collective transverse, pouvoir d'agir, approche instrumentale, cours d'action

Keywords: service relations, cross-functional collective activity, power to act, instrumental approach, course of action

\section{AUTHORS}

\section{CÉLINE PORET}

Université Paris 8 - EDF R\&D - poret_celine@yahoo.fr

\section{VIVIANE FOLCHER}

Université Paris 8 - viviane.folcher@univ-paris8.fr

FLORENCE MOTTÉ

EDF R\&D - florence.motte@edf.fr

YVON HARADJI

EDF R\&D - yvon.haradji@edf.fr 